\title{
THE ABSTRACT HODGE-DIRAC OPERATOR AND ITS STABLE DISCRETIZATION
}

\author{
PAUL LEOPARDI AND ARI STERN
}

\begin{abstract}
This paper adapts the techniques of finite element exterior calculus to study and discretize the abstract Hodge-Dirac operator, which is a square root of the abstract Hodge-Laplace operator considered by Arnold, Falk, and Winther [Bull. Amer. Math. Soc. 47 (2010), 281-354]. Dirac-type operators are central to the field of Clifford analysis, where recently there has been considerable interest in their discretization. We prove a priori stability and convergence estimates, and show that several of the results in finite element exterior calculus can be recovered as corollaries of these new estimates.
\end{abstract}

\section{INTRODUCTION}

1.1. Overview. In the numerical analysis of elliptic PDEs, much attention has been given (quite rightly) to the discretization of the second-order Laplace operator. The development of mixed finite elements (e.g., edge elements) paved the way for the discretization of other Laplace-type second-order differential operators, such as the vector Laplacian, with important numerical applications in computational electromagnetics and elasticity. The recent development of finite element exterior calculus (Arnold, Falk, and Winther [2, 3], extending earlier seminal work by Bossavit [9, 10], Hiptmair [27, 28], Kotiuga [29], Nédélec [32, 33], and many others) has shown that these operators are special cases of the Hodge-Laplace operator on differential $k$ forms, which can be stably discretized by certain families of finite element differential forms. An even more general operator, called the abstract Hodge-Laplace operator, includes both the aforementioned Hodge-Laplace operator on $k$-forms, as well as other operators that arise, for example, in elasticity.

By comparison, Dirac-type operators have received little attention from the perspective of numerical PDEs - despite being, in many ways, just as fundamental as the widely-studied Laplace operators discussed above. Informally, a Dirac operator is a square root of some Laplace operator, and is therefore a first-order (rather than second-order) differential operator. Dirac-type operators arise both in analysis (Eastwood and Ryan [20]) and in differential geometry (Friedrich [25]), in addition to their well-known, eponymous origins in quantum mechanics (Dirac [19]). The study of these first-order operators is also associated with a number of celebrated theorems, including the Atiyah-Singer index theorem [4, Witten's proof of the positive energy theorem [34], and the solution of the Kato square root problem (Axelsson et al. [5]). Clifford analysis is the study of Dirac operators in various settings, including on smooth manifolds (Delanghe [17], Cnops [16]).

Recently, there has been growing interest in developing a theory of discrete Clifford analysis, based on lattice discretizations of Dirac operators (Faustino et al. [23], Faustino [22], Brackx et al. [11]). In many respects, this work resembles the various

2010 Mathematics Subject Classification. Primary: 65N30; Secondary: 58A14.

Key words and phrases. Hodge-Dirac operator, Clifford analysis, geometric calculus, finite element exterior calculus, Hodge theory. 
lattice approaches to discretizing exterior calculus (Desbrun et al. [18, Harrison [26], Bochev and Hyman [8]), particularly in the use of primal-dual mesh pairs. These approaches are closer in spirit to finite difference methods than to finite element methods, in that they focus more on the degrees of freedom themselves than on basis functions and interpolants. Consequently, these methods tend to be less amenable to stability and convergence analysis, or to higher-order discretizations, compared with the finite element exterior calculus of Arnold et al. [2, 3].

However, scant attention has been given to the possibility of using a mixed finite element approach to discretize Dirac-type operators and their associated first-order PDEs. The present paper aims to fill this gap.

The paper is organized as follows:

(1) In the remainder of this section, we briefly provide some background on the relationship between exterior calculus and Clifford analysis, which we illustrate with examples on $\mathbb{R}^{2}$ and $\mathbb{R}^{3}$.

(2) In Section 2, we develop an abstract version of Hodge theory and the HodgeDirac operator, and prove well-posedness for the associated variational problem. Following Axelsson and McIntosh [6], Axelsson et al. [5], this approach replaces the exterior derivative by an arbitrary nilpotent operator on a Hilbert space. Despite the simplicity of this setup - even simpler than the Hilbert complexes considered by Brüning and Lesch [13] and Arnold et al. 3] -it retains all of the salient features needed for the analysis, including abstract versions of the Hodge decomposition and Poincaré inequality.

(3) In Section 3, we consider the discretization of the variational problem for the abstract Hodge-Dirac operator, proving stability and a priori error estimates. Moreover, given some additional assumptions on the nilpotent operator, we develop improved estimates based on a duality $/ L^{2}$-lifting/Aubin-Nitsche-type argument.

(4) In Section 4, we relate the abstract Hodge-Dirac operator to its square, the abstract Hodge-Laplace operator, and show that some of the key results of finite element exterior calculus (cf. Arnold et al. 3]) are recovered as corollaries of the estimates in Section 2 and Section 3.

(5) In Section 5, we briefly discuss the correspondence between the nilpotent operator formalism, considered here, and the Hilbert complex formalism used by Arnold et al. [3]. In particular, this correspondence implies that the elements used in finite element exterior calculus, e.g., the $\mathcal{P}_{r}$ and $\mathcal{P}_{r}^{-}$families of piecewise-polynomial differential forms on simplicial meshes and the $\mathcal{S}_{r}$ family on cubical meshes (cf. Arnold and Awanou [1]), also yield a stable discretization of the Hodge-Dirac problem.

(6) Finally, in Section 6, we provide a numerical application for the discretization of the Hodge-Dirac operator: computing a vector field with prescribed divergence and curl.

1.2. Motivating examples: exterior calculus and Clifford analysis. In this section, we illustrate the relationship between the Euclidean Hodge-Dirac operator for differential forms and certain Dirac operators for Clifford algebra-valued functions. In particular, we focus on the important examples of $\mathbb{R}^{2}$ and $\mathbb{R}^{3}$, where the Clifford algebra (or its even subalgebra) can be identified with the complex numbers $\mathbb{C}$ or the quaternions $\mathbb{H}$.

Example 1.1 ( $\mathbb{R}^{2}$ and the Cauchy-Riemann equations). Let $\Omega\left(\mathbb{R}^{2}\right)=\bigoplus_{k=0}^{2} \Omega^{k}\left(\mathbb{R}^{2}\right)$ denote the graded vector space of smooth differential forms on $\mathbb{R}^{2}$, where $\Omega^{k}\left(\mathbb{R}^{2}\right)$ 
denotes the subspace of $k$-forms for $k=0,1,2$. A general element $u \in \Omega\left(\mathbb{R}^{2}\right)$ has the form

$$
u=u_{0}+u_{1} \mathrm{~d} x_{1}+u_{2} \mathrm{~d} x_{2}+u_{12} \mathrm{~d} x_{1} \wedge \mathrm{d} x_{2} .
$$

Letting $\mathrm{d}$ be the (+1-graded) exterior derivative on $\Omega\left(\mathbb{R}^{2}\right)$ and $\mathrm{d}^{*}$ be its (-1-graded) $L^{2}$-adjoint, we have

$$
\begin{aligned}
\mathrm{d} u & =\partial_{1} u_{0} \mathrm{~d} x_{1}+\partial_{2} u_{0} \mathrm{~d} x_{2}+\left(\partial_{1} u_{2}-\partial_{2} u_{1}\right) \mathrm{d} x_{1} \wedge \mathrm{d} x_{2}, \\
\mathrm{~d}^{*} u & =-\left(\partial_{1} u_{1}+\partial_{2} u_{2}\right)+\partial_{2} u_{12} \mathrm{~d} x_{1}-\partial_{1} u_{12} \mathrm{~d} x_{2} .
\end{aligned}
$$

The Hodge-Dirac operator (or Hodge-de Rham operator) on $\Omega\left(\mathbb{R}^{2}\right)$ is defined by $\mathrm{D}=\mathrm{d}+\mathrm{d}^{*}$, so that

$$
\begin{aligned}
\mathrm{D} u=-\left(\partial_{1} u_{1}+\partial_{2} u_{2}\right)+\left(\partial_{1} u_{0}+\partial_{2} u_{12}\right) \mathrm{d} x_{1}+\left(\partial_{2} u_{0}\right. & \left.-\partial_{1} u_{12}\right) \mathrm{d} x_{2} \\
& +\left(\partial_{1} u_{2}-\partial_{2} u_{1}\right) \mathrm{d} x_{1} \wedge \mathrm{d} x_{2} .
\end{aligned}
$$

Note that $\mathrm{D}$ is not a graded operator with respect to the integer grading of $\Omega\left(\mathbb{R}^{2}\right)$, since $\mathrm{d}$ is +1 -graded and $\mathrm{d}^{*}$ is -1 -graded. However, if we introduce the $\mathbb{Z}_{2}$ grading $\Omega\left(\mathbb{R}^{2}\right)=\Omega^{+}\left(\mathbb{R}^{2}\right) \oplus \Omega^{-}\left(\mathbb{R}^{2}\right)$, where

$$
\begin{aligned}
& \Omega^{+}\left(\mathbb{R}^{2}\right)=\bigoplus_{k \text { even }} \Omega^{k}\left(\mathbb{R}^{2}\right)=\Omega^{0}\left(\mathbb{R}^{2}\right) \oplus \Omega^{2}\left(\mathbb{R}^{2}\right), \\
& \Omega^{-}\left(\mathbb{R}^{2}\right)=\bigoplus_{k \text { odd }} \Omega^{k}\left(\mathbb{R}^{2}\right)=\Omega^{1}\left(\mathbb{R}^{2}\right)
\end{aligned}
$$

then $\mathrm{D}$ is an odd-graded operator, mapping $\Omega^{+}\left(\mathbb{R}^{2}\right)$ to $\Omega^{-}\left(\mathbb{R}^{2}\right)$ and vice versa. Restricting the Hodge-Dirac operator to the even-degree part, whose elements are $u^{+}=u_{0}+u_{12} \mathrm{~d} x_{1} \wedge \mathrm{d} x_{2} \in \Omega^{+}\left(\mathbb{R}^{2}\right)$, we obtain

$$
\mathrm{D} u^{+}=\left(\partial_{1} u_{0}+\partial_{2} u_{12}\right) \mathrm{d} x_{1}+\left(\partial_{2} u_{0}-\partial_{1} u_{12}\right) \mathrm{d} x_{2} .
$$

Taking $i=-\mathrm{d} x_{1} \wedge \mathrm{d} x_{2}$, we can thus identify $u^{+}$with the complex-valued function $f=u_{0}-u_{12} i$, and it follows that $\mathrm{D} u^{+}=0$ if and only if

$$
\partial_{1} u_{0}+\partial_{2} u_{12}=0, \quad \partial_{2} u_{0}-\partial_{1} u_{12}=0,
$$

i.e., $f$ is a solution to the Cauchy-Riemann system.

These observations have direct analogs in the language of Clifford analysis. The Clifford algebra $\mathrm{Cliff}_{0,2}(\mathbb{R})$ on $\mathbb{R}^{2}$ has elements of the form

$$
a=a_{0}+a_{1} e_{1}+a_{2} e_{2}+a_{12} e_{1} e_{2},
$$

subject to the algebraic relations $e_{1}^{2}=e_{2}^{2}=-1$ and $e_{1} e_{2}=-e_{2} e_{1}$. This is isomorphic to the algebra of quaternions $\mathbb{H}$, while the even subalgebra $\mathrm{Cliff}_{0,2}^{+}(\mathbb{R})$, containing only even-degree terms $a^{+}=a_{0}+a_{12} e_{1} e_{2}$, is isomorphic to $\mathbb{C}$ by taking $i=-e_{1} e_{2}$. Hence, $\Omega\left(\mathbb{R}^{2}\right)$ can be identified with $\operatorname{Cliff}_{0,2}(\mathbb{R})$-valued (i.e., quaternion-valued) functions on $\mathbb{R}^{2}$, by mapping $u \mapsto u_{0}+u_{1} e_{1}+u_{2} e_{2}+u_{12} e_{1} e_{2}$. Likewise, $\Omega^{+}\left(\mathbb{R}^{2}\right)$ can be identified with $\operatorname{Cliff}_{0,2}^{+}(\mathbb{R})$-valued (i.e., complex-valued) functions on $\mathbb{R}^{2}$. Finally, we note that the Hodge-Dirac operator corresponds to the usual Dirac operator $e_{1} \partial_{1}+e_{2} \partial_{2}$ for Cliff $_{0,2}(\mathbb{R})$-valued functions, since

$$
\begin{aligned}
\left(e_{1} \partial_{1}+e_{2} \partial_{2}\right)\left(u_{0}+\right. & \left.u_{1} e_{1}+u_{2} e_{2}+u_{12} e_{1} e_{2}\right)=-\left(\partial_{1} u_{1}+\partial_{2} u_{2}\right) \\
& +\left(\partial_{1} u_{0}+\partial_{2} u_{12}\right) e_{1}+\left(\partial_{2} u_{0}-\partial_{1} u_{12}\right) e_{2}+\left(\partial_{1} u_{2}-\partial_{2} u_{1}\right) e_{1} e_{2}
\end{aligned}
$$

which agrees with the previous expression for $\mathrm{D} u$. 
Example 1.2 ( $\mathbb{R}^{3}$ and vector calculus). A general element $u \in \Omega\left(\mathbb{R}^{3}\right)$ has the form

$$
\begin{aligned}
u=u_{0}+u_{1} \mathrm{~d} x_{1} & +u_{2} \mathrm{~d} x_{2}+u_{3} \mathrm{~d} x_{3} \\
& +u_{23} \mathrm{~d} x_{2} \wedge \mathrm{d} x_{3}+u_{31} \mathrm{~d} x_{3} \wedge \mathrm{d} x_{1}+u_{12} \mathrm{~d} x_{1} \wedge \mathrm{d} x_{2} \\
& +u_{123} \mathrm{~d} x_{1} \wedge \mathrm{d} x_{2} \wedge \mathrm{d} x_{3} .
\end{aligned}
$$

Defining the odd vector field $\mathbf{u}^{-}=\left(u_{1}, u_{2}, u_{3}\right)$ and even vector field $\mathbf{u}^{+}=\left(u_{23}, u_{31}, u_{12}\right)$, the element $u$ can be written in the simpler form

$$
u=u_{0}+\mathbf{u}^{-} \cdot \mathrm{d} \mathbf{l}+\mathbf{u}^{+} \cdot \mathrm{d} \mathbf{S}+u_{123} \mathrm{~d} V .
$$

This notation is evocative of the fact that 1-, 2-, and 3-forms correspond, respectively, to line, surface, and volume integrals in vector calculus. The exterior derivative and its dual are then given by

$$
\begin{aligned}
\mathrm{d} u & =\operatorname{grad} u_{0} \cdot \mathrm{d} \mathbf{l}+\operatorname{curl} \mathbf{u}^{-} \cdot \mathrm{d} \mathbf{S}+\operatorname{div} \mathbf{u}^{+} \mathrm{d} V, \\
\mathrm{~d}^{*} u & =-\operatorname{div} \mathbf{u}^{-}+\operatorname{curl} \mathbf{u}^{+} \cdot \mathrm{d} \mathbf{l}-\operatorname{grad} u_{123} \cdot \mathrm{d} \mathbf{S},
\end{aligned}
$$

so the Hodge-Dirac operator $\mathrm{D}=\mathrm{d}+\mathrm{d}^{*}$ is defined by

$$
\mathrm{D} u=-\operatorname{div} \mathbf{u}^{-}+\left(\operatorname{grad} u_{0}+\operatorname{curl} \mathbf{u}^{+}\right) \cdot \mathrm{d} \mathbf{l}+\left(\operatorname{curl} \mathbf{u}^{-}-\operatorname{grad} u_{123}\right) \cdot \mathrm{d} \mathbf{S}+\operatorname{div} \mathbf{u}^{+} \mathrm{d} V .
$$

In particular, restricting to the even part $u^{+}=u_{0}+\mathbf{u}^{+} \cdot \mathrm{d} \mathbf{S}$ yields

$$
\mathrm{D} u^{+}=\left(\operatorname{grad} u_{0}+\operatorname{curl} \mathbf{u}^{+}\right) \cdot \mathrm{d} \mathbf{l}+\operatorname{div} \mathbf{u}^{+} \mathrm{d} V .
$$

Identifying $u^{+}$with the quaternion-valued function $f=u_{0}+u_{23} i+u_{31} j+u_{12} k$, it follows that $\mathrm{D} u^{+}=0$ if and only if

$$
\operatorname{grad} u_{0}+\operatorname{curl} \mathbf{u}^{+}=0, \quad \operatorname{div} \mathbf{u}^{+}=0,
$$

i.e., $f$ is a solution to the so-called Moisil-Théodorescu system [31], a CauchyRiemann-type system arising in quaternionic analysis.

In the language of Clifford analysis, the algebra $\mathrm{Cliff}_{0,3}(\mathbb{R})$ on $\mathbb{R}^{3}$ has elements of the form

$$
a=a_{0}+a_{1} e_{1}+a_{2} e_{2}+a_{3} e_{3}+a_{23} e_{2} e_{3}+a_{31} e_{3} e_{1}+a_{12} e_{1} e_{2}+a_{123} e_{1} e_{2} e_{3},
$$

subject to the algebraic relations $e_{1}^{2}=e_{2}^{2}=e_{3}^{2}=-1$, along with the anticommutativity relations $e_{1} e_{2}=-e_{2} e_{1}, e_{1} e_{3}=-e_{3} e_{1}$, and $e_{2} e_{3}=-e_{3} e_{2}$. This is isomorphic to the algebra of so-called split-biquaternions $\mathbb{H} \oplus \mathbb{H}$, while the even subalgebra $\mathrm{Cliff}_{0,3}^{+}(\mathbb{R})$ is isomorphic to $\mathbb{H}$. As in the previous example, there is a bijective correspondence between $\operatorname{Cliff}_{0,3}(\mathbb{R})$-valued (resp., Cliff $0_{0,3}^{+}(\mathbb{R})$-valued) functions and $\Omega\left(\mathbb{R}^{3}\right)$ (resp., $\Omega^{+}\left(\mathbb{R}^{3}\right)$ ), whereby the Dirac operator corresponds to the Hodge-Dirac operator.

\section{Hodge-Dirac theory For nilpotent operators}

In this section, we develop an abstract version of Hodge theory, where the exterior derivative is replaced by an arbitrary nilpotent operator on a Hilbert space. This abstraction has appeared earlier, notably in the papers Axelsson and McIntosh [6], Axelsson et al. [5]. After introducing this basic machinery, we study an abstract Hodge-Dirac operator and its associated variational problem, for which we prove a well-posedness result. 


\subsection{Nilpotent operators and the abstract Hodge decomposition.}

Definition 2.1. Let d be a closed, densely-defined linear operator on a (real) Hilbert space $W$, with domain $\mathcal{D}(\mathrm{d})$, range $\mathcal{R}(\mathrm{d})$, and kernel $\mathcal{N}(\mathrm{d})$. Then we say that $\mathrm{d}$ is

(i) nilpotent if $\mathcal{R}(\mathrm{d}) \subset \mathcal{N}(\mathrm{d})$, so that $\mathrm{d}^{2}=0$;

(ii) closed-nilpotent if, in addition to (i), $\mathcal{R}(\mathrm{d})$ is closed;

(iii) Fredholm-nilpotent if, in addition to (i)-(ii), $\mathcal{R}(\mathrm{d})$ has finite codimension in $\mathcal{N}(\mathrm{d})$

(iv) diffuse Fredholm-nilpotent if, in addition to (i)-(iii), $\mathcal{D}(\mathrm{d}) \cap \mathcal{N}(\mathrm{d})^{\perp}$ is compact in $\mathcal{N}(\mathrm{d})^{\perp}$.

Given a nilpotent operator d on a Hilbert space $W$, define the subspaces

$$
\mathfrak{Z}=\mathcal{N}(\mathrm{d}), \quad \mathfrak{B}=\mathcal{R}(\mathrm{d}) \subset \mathfrak{Z}, \quad \mathfrak{H}=\mathfrak{Z} \cap \mathfrak{B}^{\perp_{W}} .
$$

The notation for these spaces originates from homology theory: $\mathfrak{B}$ stands for boundary, $\mathfrak{Z}$ stands for cycle (German: Zyklus), and $\mathfrak{H}$ stands for harmonic. It follows immediately that $W$ has the orthogonal decomposition

$$
W=\mathfrak{Z} \oplus \mathfrak{Z}^{\perp_{W}}=\mathfrak{Z} \cap\left(\overline{\mathfrak{B}} \oplus \mathfrak{B}^{\perp_{W}}\right) \oplus \mathfrak{Z}^{\perp_{W}}=\overline{\mathfrak{B}} \oplus \mathfrak{H} \oplus \mathfrak{Z}^{\perp_{W}},
$$

which we call the abstract Hodge decomposition of $W$. The adjoint $\mathrm{d}^{*}$ is also a nilpotent operator, so we can define the corresponding subspaces $\mathfrak{Z}^{*}=\mathcal{N}\left(\mathrm{d}^{*}\right)$ and $\mathfrak{B}^{*}=\mathcal{R}\left(\mathrm{d}^{*}\right) \subset \mathfrak{Z}^{*}$. However, since $\mathrm{d}$ and $\mathrm{d}^{*}$ are adjoints, it is possible to write

$$
\mathfrak{Z}^{*}=\mathfrak{B}^{\perp_{W}}, \quad \overline{\mathfrak{B}^{*}}=\mathfrak{Z}^{\perp_{W}}, \quad \mathfrak{H}=\mathfrak{Z} \cap \mathfrak{Z}^{*} .
$$

Hence, the Hodge decomposition has the alternative form,

$$
W=\overline{\mathfrak{B}} \oplus \mathfrak{H} \oplus \overline{\mathfrak{B}^{*}} .
$$

When $\mathrm{d}$ is closed-nilpotent, so is $\mathrm{d}^{*}$, and so $\mathfrak{B}$ and $\mathfrak{B}^{*}$ are closed subspaces. In this case, the Hodge decomposition becomes

$$
W=\mathfrak{B} \oplus \mathfrak{H} \oplus \mathfrak{Z}^{\perp_{W}}=\mathfrak{B} \oplus \mathfrak{H} \oplus \mathfrak{B}^{*} .
$$

Finally, note that $d$ is (diffuse) Fredholm-nilpotent if and only if $\mathrm{d}^{*}$ is.

The operator d is generally unbounded on $W$. However, we can equip the dense domain $V=\mathcal{D}(\mathrm{d}) \subset W$ with its own Hilbert space structure, so that $\mathrm{d}$ is a bounded operator on $V$. Denoting the inner product on $W$ by $\langle\cdot, \cdot\rangle$, let $V$ be endowed with the graph inner product,

$$
\langle u, v\rangle_{V}=\langle u, v\rangle+\langle\mathrm{d} u, \mathrm{~d} v\rangle, \quad \forall u, v \in V .
$$

Since $\mathrm{d}$ is a closed operator, its graph is closed, and hence $V$ is complete with respect to the norm $\|\cdot\|_{V}$ induced by the inner product. The operator $\mathrm{d}$ is bounded (in fact, nonexpansive) with respect to the $V$-norm, since

$$
\|\mathrm{d} v\|_{V}^{2}=\|\mathrm{d} v\|^{2} \leq\|v\|^{2}+\|\mathrm{d} v\|^{2}=\|v\|_{V}^{2} .
$$

Since $\mathfrak{B}$ and $\mathfrak{Z}$ are both in $V$, we again obtain an abstract Hodge decomposition,

$$
V=\overline{\mathfrak{B}} \oplus \mathfrak{H} \oplus \mathfrak{Z}^{\perp},
$$

where $\mathfrak{Z}^{\perp}=\mathfrak{Z}^{\perp_{W}} \cap V$. Finally, d is closed- or Fredholm-nilpotent on $V$ if and only if it is on $W$, and in this case we have

$$
V=\mathfrak{B} \oplus \mathfrak{H} \oplus \mathfrak{Z}^{\perp},
$$

as before. Given $v \in V$, we denote the components of its Hodge decomposition by $v=v_{\mathfrak{B}}+v_{\mathfrak{H}}+v_{\perp}$. 
The Poincaré inequality is one of the most important corollaries of the Hodge decomposition, from an analytical standpoint. This was demonstrated in Arnold et al. [3] in the abstract setting of closed Hilbert complexes; in the special case of the de Rham complex, one obtains the classical Poincaré inequality. By the same argument, one also obtains an abstract Poincaré inequality for closed-nilpotent operators.

Lemma 2.2 (Poincaré inequality). If $\mathrm{d}$ is a closed-nilpotent operator, then there exists a constant $c_{P} \geq 1$, called the Poincaré constant, such that

$$
\|v\|_{V} \leq c_{P}\|\mathrm{~d} v\|, \quad \forall v \in \mathfrak{Z}^{\perp} .
$$

Proof. The linear map d restricts to a bounded bijection between $\mathfrak{Z}^{\perp}$ and $\mathfrak{B}$, both of which are closed subspaces of $V$. Hence, Banach's bounded inverse theorem (a standard corollary of the open mapping theorem) implies that $\left.\mathrm{d}\right|_{\mathfrak{Z}^{\perp}}$ has a bounded inverse, which proves the result. (The bound $c_{P} \geq 1$ is a result of the nonexpansiveness of d with respect to the $V$ norm.)

2.2. The abstract Hodge-Dirac operator. Having defined abstract versions of the exterior derivative and Hodge decomposition, we are now prepared to define the abstract Hodge-Dirac operator.

Definition 2.3. Given a Hilbert space $W$ with a nilpotent operator $\mathrm{d}$ and adjoint $\mathrm{d}^{*}$, the abstract Hodge-Dirac operator is $\mathrm{D}=\mathrm{d}+\mathrm{d}^{*}$.

The Hodge-Dirac operator inherits several of the properties of $d$ and $d^{*}$. Like those operators, it is closed and densely-defined, and its domain, range, and kernel are given by

$$
\mathcal{D}(\mathrm{D})=\mathcal{D}(\mathrm{d}) \cap \mathcal{D}\left(\mathrm{d}^{*}\right), \quad \mathcal{R}(\mathrm{D})=\mathfrak{B} \oplus \mathfrak{B}^{*}, \quad \mathcal{N}(\mathrm{D})=\mathfrak{Z} \cap \mathfrak{Z}^{*}=\mathfrak{H} .
$$

Moreover, D has closed range if and only if $\mathrm{d}$ is closed-nilpotent, and is (diffuse) Fredholm if and only if $\mathrm{d}$ is (diffuse) Fredholm-nilpotent (cf. Axelsson and McIntosh [6. Propositions 3.5 and 3.11]). Unlike $\mathrm{d}$ and $\mathrm{d}^{*}$, though, $\mathrm{D}$ is self-adjoint and is not nilpotent; in fact, its square is

$$
\mathrm{D}^{2}=\mathrm{dd}^{*}+\mathrm{d}^{*} \mathrm{~d}=L,
$$

which is called the abstract Hodge-Laplace operator.

The Hodge decomposition implies that $W=\overline{\mathcal{R}(\mathrm{D})} \oplus \mathcal{N}(\mathrm{D})$. In particular, when $\mathrm{d}$ is closed-nilpotent, $\mathcal{R}(\mathrm{D})$ is closed, so the Hodge decomposition is $W=\mathcal{R}(\mathrm{D}) \oplus \mathcal{N}(\mathrm{D})$. In this latter case, the Hodge decomposition is simply an expression of the closed range theorem for the self-adjoint operator $\mathrm{D}$. This decomposition makes it natural to pose the following problem: Given $f \in W$, find $(u, p) \in\left(\mathcal{D}(D) \cap \mathcal{N}(\mathrm{D})^{\perp}\right) \oplus \mathcal{N}(\mathrm{D})$ satisfying

$$
\mathrm{D} u+p=f .
$$

The solution to this problem gives the Hodge decomposition $f=\mathrm{d} u+\mathrm{d}^{*} u+p$.

We now consider the associated variational problem: Find $(u, p) \in V \times \mathfrak{H}$ such that

$$
\begin{aligned}
\langle\mathrm{d} u, v\rangle+\langle u, \mathrm{~d} v\rangle+\langle p, v\rangle & =\langle f, v\rangle, & & \forall v \in V, \\
\langle u, q\rangle & =0, & & \forall q \in \mathfrak{H} .
\end{aligned}
$$

If we define the bilinear form $B:(V \times \mathfrak{H}) \times(V \times \mathfrak{H}) \rightarrow \mathbb{R}$ to be

$$
B(u, p ; v, q)=\langle\mathrm{d} u, v\rangle+\langle u, \mathrm{~d} v\rangle+\langle p, v\rangle+\langle u, q\rangle,
$$


then the variational problem can be rewritten as: Find $(u, p) \in V \times \mathfrak{H}$ such that

$$
B(u, p ; v, q)=\langle f, v\rangle, \quad \forall(v, q) \in V \times \mathfrak{H} .
$$

Note that $B$ is bounded (by a straightforward application of the Cauchy-Schwarz inequality) and symmetric. Therefore, in order to establish the well-posedness of this problem, it suffices to prove the "inf-sup condition"

$$
\gamma=\inf _{(0,0) \neq(u, p) \in V \times \mathfrak{H}(0,0) \neq(v, q) \in V \times \mathfrak{H}} \sup _{\|(u, p)\|_{V \times \mathfrak{H}}\|(v, q)\|_{V \times \mathfrak{H}}}>0,
$$

which is implied by the following theorem. (Compare Arnold et al. [3, Theorem 3.2].)

Theorem 2.4. Suppose $\mathrm{d}$ is a closed-nilpotent operator on a Hilbert space $W$ with dense domain $V \subset W$. Then there exists a constant $\gamma>0$, depending only on the Poincaré constant $c_{P}$, such that for all nonzero $(u, p) \in V \times \mathfrak{H}$, there exists a nonzero $(v, q) \in V \times \mathfrak{H}$ satisfying

$$
B(u, p ; v, q) \geq \gamma\left(\|u\|_{V}+\|p\|\right)\left(\|v\|_{V}+\|q\|\right) .
$$

Notation. In the following proof, and in the remainder of this paper, we follow the common practice of letting $C$ denote an unspecified, positive constant, whose value may differ with each occurrence (even within the same proof).

Proof. Take the test functions

$$
v=\rho+p+\mathrm{d} u, \quad q=u_{\mathfrak{H}},
$$

where $\rho \in \mathfrak{Z}^{\perp}$ is the unique element such that $\mathrm{d} \rho=u_{\mathfrak{B}}$. Using the Poincaré inequality and the orthogonality of the Hodge decomposition, observe that

$$
\begin{aligned}
\|v\|_{V}+\|q\| & \leq\|\rho\|_{V}+\|p\|+\|\mathrm{d} u\|+\left\|u_{\mathfrak{H}}\right\| \\
& \leq c_{P}\left\|u_{\mathfrak{B}}\right\|+\left\|u_{\mathfrak{H}}\right\|+\|\mathrm{d} u\|+\|p\| \\
& \leq C\left(\|u\|_{V}+\|p\|\right) .
\end{aligned}
$$

Next, substituting these test functions into the bilinear form,

$$
\begin{aligned}
B(u, p ; v, q) & =\|\mathrm{d} u\|^{2}+\left\langle u, u_{\mathfrak{B}}\right\rangle+\|p\|^{2}+\left\langle u, u_{\mathfrak{H}}\right\rangle \\
& =\|\mathrm{d} u\|^{2}+\left\|u_{\mathfrak{B}}\right\|^{2}+\left\|u_{\mathfrak{H}}\right\|^{2}+\|p\|^{2} \\
& =\frac{1}{2}\|\mathrm{~d} u\|^{2}+\frac{1}{2}\left\|\mathrm{~d} u_{\perp}\right\|^{2}+\left\|u_{\mathfrak{B}}\right\|^{2}+\left\|u_{\mathfrak{H}}\right\|^{2}+\|p\|^{2} \\
& \geq \frac{1}{2}\|\mathrm{~d} u\|^{2}+\frac{1}{2 c_{P}^{2}}\left\|u_{\perp}\right\|^{2}+\left\|u_{\mathfrak{B}}\right\|^{2}+\left\|u_{\mathfrak{H}}\right\|^{2}+\|p\|^{2} \\
& \geq \frac{1}{2 c_{P}^{2}}\left(\|u\|_{V}^{2}+\|p\|^{2}\right),
\end{aligned}
$$

where the last inequality follows from $c_{P} \geq 1$ and the Hodge decomposition. Combining this with the previous inequality, we therefore obtain

$$
B(u, p ; v, q) \geq \gamma\left(\|u\|_{V}+\|p\|\right)\left(\|v\|_{V}+\|q\|\right),
$$

as claimed.

Corollary 2.5. The variational problem for the abstract Hodge-Dirac operator is well-posed. That is, there exists a constant c, depending only on the Poincaré constant $c_{P}$, such that for all $f \in W$, the problem (1) has a unique solution $(u, p) \in V \times \mathfrak{H}$, which satisfies the estimate

$$
\|u\|_{V}+\|p\| \leq c\|f\|
$$


Proof. This follows directly from the inf-sup condition, cf. Babuška [7].

\section{Numerical Stability AND CONVERGEnCE of A DisCRETE PROBlem}

In this section, we discuss the approximation of the Hodge-Dirac variational problem (1) on a closed subspace $V_{h} \subset V$. We will often refer to this as the discrete Hodge-Dirac problem, since $V_{h}$ is typically a finite-dimensional subspace obtained by some discretization process, e.g., finite-element discretization. First, in Section 3.1, we discuss some additional structure that must be assumed - crucially, as in Arnold et al. [3], we require the existence of a bounded projection, which along with the inclusion map $V_{h} \hookrightarrow V$ must commute with the differentials - and the consequences of this additional structure. Next, in Section 3.2, we introduce the discrete problem and prove stability and convergence estimates. Finally, in Section 3.3, we give improved estimates for the case where $d$ is not merely closed-nilpotent but diffuse Fredholm-nilpotent.

\subsection{Approximation by a subspace with a bounded commuting projection.}

Let $V_{h} \subset V$ be a closed (e.g., finite-dimensional) subspace of $V$, such that $\mathrm{d} V_{h} \subset V_{h}$. If $\mathrm{d}$ is closed-nilpotent on $V$, then the restriction $\mathrm{d}_{h}=\left.\mathrm{d}\right|_{V_{h}}$ is closed-nilpotent on $V_{h}$, and this induces an abstract Hodge decomposition $V_{h}=\mathfrak{B}_{h} \oplus \mathfrak{H}_{h} \oplus \mathfrak{Z}_{h}^{\perp}$. Note that, although $\mathrm{d}_{h}$ is the restriction of $\mathrm{d}$ to $V_{h}$, its adjoint $\mathrm{d}_{h}^{*}$ with respect to the $W$-inner product is generally not the restriction of $\mathrm{d}^{*}$; consequently, we have $\mathfrak{B}_{h} \subset \mathfrak{B}$ and $\mathfrak{Z}_{h} \subset \mathfrak{Z}$, but generally $\mathfrak{H}_{h} \not \subset \mathfrak{H}$ and $\mathfrak{Z}_{h}^{\perp} \not \subset \mathfrak{Z}^{\perp}$.

We now make one additional assumption: suppose also that there exists a bounded projection $\pi_{h} \in \mathcal{L}\left(V, V_{h}\right)$ such that $\pi_{h} \mathrm{~d} v=\mathrm{d} \pi_{h} v$ for all $v \in V$. (By "projection," we mean only that $\pi_{h}$ is idempotent and surjective onto $V_{h}$, not that it is an orthogonal projection.) It is nontrivial to show that such projections exist, so their explicit construction for the Hodge-de Rham complex (cf. Christiansen and Winther [14, Falk and Winther [21]) was a major technical advance in finite element exterior calculus. The importance of this assumption is that it allows us to control the Poincaré constant of $V_{h}$ in terms of $c_{P}$ and $\left\|\pi_{h}\right\|$, as shown in the following lemma (essentially similar to Arnold et al. [3, Theorem 3.6]).

Lemma 3.1. Let $\mathrm{d}$ be a closed-nilpotent operator on a Hilbert space $W$ with dense domain $V \subset W$, and suppose that $V_{h} \subset V$ is a closed subspace with a bounded commuting projection $\pi_{h} \in \mathcal{L}\left(V, V_{h}\right)$ such that $\pi_{h} \mathrm{~d}=\mathrm{d} \pi_{h}$. Then

$$
\left\|v_{h}\right\|_{V} \leq c_{P}\left\|\pi_{h}\right\|\left\|\mathrm{d} v_{h}\right\|, \quad \forall v_{h} \in \mathfrak{Z}_{h}^{\perp} .
$$

In other words, the Poincaré constant of $V_{h}$ is bounded by $c_{P}\left\|\pi_{h}\right\|$.

Proof. Given $v_{h} \in \mathfrak{Z}_{h}^{\perp}$, let $z \in \mathfrak{Z}^{\perp}$ be the element satisfying $\mathrm{d} z=\mathrm{d} v_{h}$. This $z$ exists and is unique, since $\left.\mathrm{d}\right|_{\mathfrak{Z}^{\perp}}$ is a bijection between $\mathfrak{Z}^{\perp}$ and $\mathfrak{B} \supset \mathfrak{B}_{h} \ni \mathrm{d} v_{h}$. Moreover, by Lemma 2.2, we have

$$
\|z\|_{V} \leq c_{P}\|\mathrm{~d} z\|=c_{P}\left\|\mathrm{~d} v_{h}\right\| .
$$

Thus, it suffices to show $\left\|v_{h}\right\|_{V} \leq\left\|\pi_{h}\right\|\|z\|_{V}$. Observe that

$$
\mathrm{d} v_{h}=\pi_{h} \mathrm{~d} v_{h}=\pi_{h} \mathrm{~d} z=\mathrm{d} \pi_{h} z,
$$

which implies that $\mathrm{d}\left(v_{h}-\pi_{h} z\right)=0$. Hence, $v_{h}-\pi_{h} z \in \mathfrak{Z}_{h} \perp v_{h}$, so

$$
\left\|v_{h}\right\|_{V}^{2}=\left\langle v_{h}, v_{h}-\pi_{h} z\right\rangle_{V}+\left\langle v_{h}, \pi_{h} z\right\rangle_{V}=\left\langle v_{h}, \pi_{h} z\right\rangle_{V} \leq\left\|v_{h}\right\|_{V}\left\|\pi_{h}\right\|\|z\|_{V} .
$$

Finally, dividing through by $\left\|v_{h}\right\|_{V}$ completes the proof. 
Unlike with orthogonal projection, generally $\pi_{h} v$ is not the best approximation to $v$ in $V_{h}$. However, it is nearly as good: since $\pi_{h} v_{h}=v_{h}$ for all $v_{h} \in V_{h}$, we have

$$
\left\|v-\pi_{h} v\right\|_{V}=\left\|\left(I-\pi_{h}\right) v\right\|_{V}=\left\|\left(I-\pi_{h}\right)\left(v-v_{h}\right)\right\|_{V} \leq C\left\|v-v_{h}\right\|_{V}
$$

and therefore

$$
\left\|v-\pi_{h} v\right\|_{V} \leq C \inf _{v_{h} \in V_{h}}\left\|v-v_{h}\right\|_{V}
$$

In other words, the approximation error differs from the optimum by at most a constant factor, a property known as quasi-optimality.

3.2. Stability and convergence of the discrete problem. Since $\mathrm{d}_{h}$ is a closednilpotent operator on $V_{h}$, we may define a discrete Hodge-Dirac operator $\mathrm{D}_{h}=\mathrm{d}_{h}+\mathrm{d}_{h}^{*}$. (Note that, since $\mathrm{d}_{h}^{*}$ is generally not the restriction of $\mathrm{d}^{*}$ to $V_{h}$, neither is $\mathrm{D}_{h}$ simply the restriction of D.) The discrete version of the variational problem (1) is then: Find $\left(u_{h}, p_{h}\right) \in V_{h} \times \mathfrak{H}_{h}$ satisfying

$$
\begin{aligned}
\left\langle\mathrm{d} u_{h}, v_{h}\right\rangle+\left\langle u_{h}, \mathrm{~d} v_{h}\right\rangle+\left\langle p_{h}, v_{h}\right\rangle & =\left\langle f, v_{h}\right\rangle, & & \forall v_{h} \in V_{h}, \\
\left\langle u_{h}, q_{h}\right\rangle & =0, & & \forall q_{h} \in \mathfrak{H}_{h} .
\end{aligned}
$$

Once again, since generally $\mathfrak{H}_{h} \not \subset \mathfrak{H}$, it follows that $V_{h} \times \mathfrak{H}_{h} \not \subset V \times \mathfrak{H}$. Hence, (2) is not simply a Galerkin discretization of the continuous variational problem (1); rather, there is a "variational crime" that will need to be accounted for in the subsequent numerical analysis.

The following theorem gives a discrete inf-sup condition, thereby showing the stability of the discretization and the well-posedness of (2).

Theorem 3.2. Let $W$ be a Hilbert space with a closed-nilpotent operator d defined on the dense domain $V \subset W$. Suppose that $V_{h} \subset V$ is a closed subspace satisfying $\mathrm{d} V_{h} \subset V_{h}$ and equipped with a bounded projection $\pi_{h}: V \rightarrow V_{h}$ such that $\pi_{h} \mathrm{~d}=\mathrm{d} \pi_{h}$. Then there exists a constant $\gamma_{h}>0$, depending only on the Poincaré constant $c_{P}$ and on the norm of $\pi_{h}$, such that for all nonzero $\left(u_{h}, p_{h}\right) \in V_{h} \times \mathfrak{H}_{h}$, there exists a nonzero $\left(v_{h}, q_{h}\right) \in V_{h} \times \mathfrak{H}_{h}$ satisfying

$$
B\left(u_{h}, p_{h} ; v_{h}, q_{h}\right) \geq \gamma_{h}\left(\left\|u_{h}\right\|_{V}+\left\|p_{h}\right\|\right)\left(\left\|v_{h}\right\|_{V}+\left\|q_{h}\right\|\right) .
$$

Proof. This follows immediately from Theorem 2.4 and Lemma 3.1.

Corollary 3.3. The discrete variational problem for the Hodge-Dirac operator is well-posed. That is, there exists a constant $c_{h}$, depending only on the Poincaré constant $c_{P}$ and on the norm of $\pi_{h}$, such that for all $f \in W$, the problem (2) has a unique solution $\left(u_{h}, p_{h}\right) \in V_{h} \times \mathfrak{H}_{h}$, which satisfies

$$
\left\|u_{h}\right\|_{V}+\left\|p_{h}\right\| \leq c_{h}\|f\| .
$$

The next theorem provides a quasi-optimal a priori error estimate for the approximation of solutions to (1) by those to (2). (Compare Arnold et al. [3, Theorem 3.9].)

Notation. In the statement of the theorem, $P_{\mathfrak{B}}$ and $P_{\mathfrak{H}}$ denote the $W$-orthogonal projections onto $\mathfrak{B}$ and $\mathfrak{H}$, respectively. We will use similar notation, throughout the remainder of the paper, to denote $W$-orthogonal projection onto these and other closed subspaces.

Theorem 3.4. Let $W$ be a Hilbert space with a closed-nilpotent operator d defined on the dense domain $V \subset W$. Suppose that $V_{h} \subset V$ is a family of closed subspaces, parametrized by $h$, satisfying $\mathrm{d} V_{h} \subset V_{h}$. Suppose also that these subspaces are equipped with projections $\pi_{h}: V \rightarrow V_{h}$, bounded uniformly in $h$, such that $\pi_{h} \mathrm{~d}=\mathrm{d} \pi_{h}$. 
If $(u, p) \in V \times \mathfrak{H}$ solves (1) and $\left(u_{h}, p_{h}\right) \in V_{h} \times \mathfrak{H}_{h}$ solves (2), then we have the error estimate

$$
\left\|u-u_{h}\right\|_{V}+\left\|p-p_{h}\right\| \leq C\left(\inf _{v \in V_{h}}\|u-v\|_{V}+\inf _{q \in V_{h}}\|p-q\|_{V}+\mu \inf _{v \in V_{h}}\left\|P_{\mathfrak{B}} u-v\right\|_{V}\right),
$$

where $\mu=\left\|\left(I-\pi_{h}\right) P_{\mathfrak{H}}\right\|$.

Proof. First, observe from the variational principles (1) and (2) that

$$
B\left(u, p ; v_{h}, q_{h}\right)=\left\langle f, v_{h}\right\rangle+\left\langle u, q_{h}\right\rangle=B\left(u_{h}, p_{h} ; v_{h}, q_{h}\right)+\left\langle u, q_{h}\right\rangle .
$$

Now, let $v$ and $q$ be the $V$-orthogonal projections of $u$ and $p$ onto $V_{h}$ and $\tilde{H}_{h}$, respectively. Then, using the previous observation and the boundedness of the bilinear form $B$, we have

$$
\begin{aligned}
B\left(u_{h}-v, p_{h}-q ; v_{h}, q_{h}\right)= & B\left(u-v, p-q ; v_{h}, q_{h}\right)-\left\langle u, q_{h}\right\rangle \\
& \leq C\left(\|u-v\|_{V}+\|p-q\|+\left\|P_{\mathfrak{H}_{h}} u\right\|\right)\left(\left\|v_{h}\right\|_{V}+\left\|q_{h}\right\|\right) .
\end{aligned}
$$

Therefore, applying the discrete inf-sup condition yields

$$
\left\|u_{h}-v\right\|_{V}+\left\|p_{h}-q\right\| \leq C\left(\|u-v\|_{V}+\|p-q\|+\left\|P_{\mathfrak{H}_{h}} u\right\|\right) .
$$

It now remains to estimate the terms $\|p-q\|$ and $\left\|P_{\mathfrak{H}_{h}} u\right\|$.

For the former term, note that $p \in \mathfrak{H} \perp \mathfrak{B} \supset \mathfrak{B}_{h}$, so

$$
P_{\mathfrak{Z}_{h}} p=P_{\mathfrak{H}_{h}} p+P_{\mathfrak{B}_{h}} p=q+0=q .
$$

On the other hand, since $p \in \mathfrak{H} \subset \mathfrak{Z}$, it follows that $\pi_{h} p \in \mathfrak{Z}_{h}$. Hence,

$$
\|p-q\|=\left\|\left(I-P_{\mathfrak{Z}_{h}}\right) p\right\| \leq\left\|\left(I-\pi_{h}\right) p\right\| \leq C \inf _{q \in V_{h}}\|p-q\|_{V},
$$

where we have used the optimality property of $P_{\mathfrak{Z}_{h}}$ and the quasi-optimality property of $\pi_{h}$. (Compare Arnold et al. [3, Theorem 3.5].)

For the latter term, we have $u \perp \mathfrak{H}$, so its Hodge decomposition can be written $u=u_{\mathfrak{B}}+u_{\perp}$. However, $u_{\perp} \perp \mathfrak{Z} \supset \mathfrak{H}_{h}$, so $P_{\mathfrak{H}_{h}} u=P_{\mathfrak{H}_{h}} u_{\mathfrak{B}}$. Furthermore, since $\pi_{h} u_{\mathfrak{B}} \in \mathfrak{B}_{h} \perp \mathfrak{H}_{h}$, we have

$$
P_{\mathfrak{H}_{h}} u=P_{\mathfrak{H}_{h}} u_{\mathfrak{B}}=P_{\mathfrak{H}_{h}}\left(u_{\mathfrak{B}}-\pi_{h} u_{\mathfrak{B}}\right)=P_{\mathfrak{H}_{h}}\left(I-\pi_{h}\right) u_{\mathfrak{B}}
$$

This implies $\left\|P_{\mathfrak{H}_{h}} u\right\|^{2}=\left\langle\left(I-\pi_{h}\right) u_{\mathfrak{B}}, P_{\mathfrak{H}_{h}} u\right\rangle$, and since $\left(I-\pi_{h}\right) u_{\mathfrak{B}} \in \mathfrak{B} \perp \mathfrak{H}$,

$$
\left\|P_{\mathfrak{H}_{h}} u\right\|^{2}=\left\langle\left(I-\pi_{h}\right) u_{\mathfrak{B}},\left(I-P_{\mathfrak{H}}\right) P_{\mathfrak{H} h} u\right\rangle \leq\left\|\left(I-\pi_{h}\right) u_{\mathfrak{B}}\right\|\left\|\left(I-P_{\mathfrak{H}}\right) P_{\mathfrak{H}_{h}} u\right\| .
$$

Next, since $P_{\mathfrak{H}_{h}} u \in \mathfrak{H}_{h} \subset \mathfrak{Z}$, we have $\left(I-P_{\mathfrak{H}}\right) P_{\mathfrak{H}_{h}} u \in \mathfrak{B}$. This implies that $\pi_{h}\left(I-P_{\mathfrak{H}}\right) P_{\mathfrak{H}_{h}} u \in \mathfrak{B}_{h}$ is orthogonal to both $P_{\mathfrak{H}_{h}} u$ and $P_{\mathfrak{H}} P_{\mathfrak{H}_{h}} u$, and hence to $\left(I-P_{\mathfrak{H}}\right) P_{\mathfrak{H}_{h}} u$, so by the Pythagorean theorem,

$$
\begin{aligned}
\left\|\left(I-P_{\mathfrak{H}}\right) P_{\mathfrak{H}_{h}} u\right\| & \leq\left\|\left(I-P_{\mathfrak{H}}\right) P_{\mathfrak{H}_{h}} u-\pi_{h}\left(I-P_{\mathfrak{H}}\right) P_{\mathfrak{H}_{h}} u\right\| \\
& =\left\|\left(I-\pi_{h}\right) P_{\mathfrak{H}} P_{\mathfrak{H}_{h}} u\right\| \\
& \leq \mu\left\|P_{\mathfrak{H}_{h}} u\right\| .
\end{aligned}
$$

Finally, combining this with the estimate above for $\left\|P_{\mathfrak{H}_{h}} u\right\|^{2}$, and using the quasioptimality property of $\pi_{h}$, we have

$$
\left\|P_{\mathfrak{H}_{h}} u\right\| \leq \mu\left\|\left(I-\pi_{h}\right) u_{\mathfrak{B}}\right\| \leq C \mu \inf _{v \in V_{h}}\left\|u_{\mathfrak{B}}-v\right\|_{V} .
$$

Altogether, it has now been shown that

$$
\left\|u_{h}-v\right\|_{V}+\left\|p_{h}-q\right\| \leq C\left(\inf _{v \in V_{h}}\|u-v\|_{V}+\inf _{q \in V_{h}}\|p-q\|_{V}+\mu \inf _{v \in V_{h}}\left\|P_{\mathfrak{B}} u-v\right\|_{V}\right)
$$


so the result follows by an application of the triangle inequality.

Finally, note that if the family of subspaces $\left\{V_{h}\right\}_{h>0}$ is pointwise approximating in $V$, in the sense that

$$
\inf _{v_{h} \in V_{h}}\left\|v-v_{h}\right\|_{V} \rightarrow 0 \text { as } h \rightarrow 0 \text { for all } v \in V
$$

then Theorem 3.4 immediately implies that $\left(u_{h}, p_{h}\right) \rightarrow(u, p)$ in $V \times V$.

3.3. Improved error estimates for diffuse Fredholm operators. In this section, we obtain improved error estimates under the stronger assumption that $d$ is not only closed-nilpotent, but is diffuse Fredholm-nilpotent, cf. Definition 2.1. The approach is related to other improved estimates obtained using duality techniques; these are known by various names, such as the "Aubin-Nitsche trick" and " $L 2$ lifting" (cf. Ciarlet [15, Theorem 3.2.4]). The proofs given here owe a considerable debt to Arnold et al. [3, Section 3.5], whose techniques for the Hodge-Laplace problem we have adapted to the Hodge-Dirac problem, with some modifications.

Assume now that $\mathrm{d}$ is a diffuse Fredholm-nilpotent operator. It follows that D is diffuse Fredholm, so the solution operator $K$ on $W$, which takes $f \mapsto u$, is compact. Moreover, $P_{\mathfrak{H}}$ is also compact, since the Fredholm property implies $\operatorname{dim} \mathfrak{H}<\infty$. Finally, we add the assumption that $\pi_{h}$ is a bounded operator on $W$, whereas previously, we had assumed only that it was bounded on $V$.

Following Arnold et al. [3], we denote $\eta=\left\|\left(I-\pi_{h}\right) K\right\|$ and, as before, $\mu=$ $\left\|\left(I-\pi_{h}\right) P_{\mathfrak{H}}\right\|$. If the family of subspaces $\left\{V_{h}\right\}_{h>0}$ is pointwise approximating in $V$, then it follows that $\left\{W_{h}\right\}_{h>0}$ is pointwise approximating in $W$, since

$$
\inf _{w_{h} \in W_{h}}\left\|w-w_{h}\right\| \leq \inf _{v \in V}\left(\|w-v\|+\inf _{v_{h} \in V_{h}}\left\|v-v_{h}\right\|_{V}\right) \rightarrow 0,
$$

by density of $V$ in $W$ together with the pointwise approximating condition in $V$. Moreover, if the operators $\pi_{h}$ are uniformly bounded in $h$, then quasi-optimality implies that $I-\pi_{h} \rightarrow 0$ pointwise in $W$. Since $K$ and $P_{\mathfrak{H}}$ are compact operators, they convert pointwise convergence to norm convergence, and therefore $\eta, \mu \rightarrow 0$. In the typical case of the de Rham complex, when $V_{h}$ consists of piecewise polynomial differential forms up to degree $r$, we will have $\eta=O(h)$ and $\mu=O\left(h^{r+1}\right.$ ) (Arnold et al. [3, p. 312]).

In Theorem 3.4, recall that we bounded the quantity $\left\|u-u_{h}\right\|_{V}+\left\|p-p_{h}\right\|$. Refined estimates will now be obtained by breaking this up into several components,

$$
\left\|\mathrm{d}\left(u-u_{h}\right)\right\|, \quad\left\|P_{\mathfrak{B}} u-P_{\mathfrak{B}_{h}} u_{h}\right\|, \quad\left\|P_{\mathfrak{B}^{*}} u-P_{\mathfrak{B}_{h}^{*}} u_{h}\right\|, \quad\left\|p-p_{h}\right\|,
$$

and estimating each of these individually, in a sequence of theorems. Equivalently, these results can be interpreted as giving error estimates for the individual terms of the discrete Hodge decomposition. Before doing so, we begin with a lemma that will be useful throughout this section.

Lemma 3.5. If $v_{h} \in \mathfrak{Z}_{h}^{\perp}$ and $v=P_{\mathfrak{B}^{*}} v_{h}$, then

$$
\left\|v-v_{h}\right\| \leq\left\|\left(I-\pi_{h}\right) v\right\| \leq \eta\left\|\mathrm{d} v_{h}\right\| .
$$

Proof. Since $\pi_{h} v-v_{h}=\pi_{h}\left(v-v_{h}\right) \in \mathfrak{Z}_{h} \subset \mathfrak{Z}$, we have $\left(v_{h}-\pi_{h} v\right) \perp\left(v_{h}-v\right)$. Therefore, the Pythagorean theorem implies

$$
\left\|v-v_{h}\right\| \leq\left\|v-\pi_{h} v\right\|=\left\|\left(I-\pi_{h}\right) v\right\| .
$$

Finally, observing that $P_{\mathfrak{B}^{*}}=K \mathrm{~d}$, it follows that $v=K \mathrm{~d} v_{h}$, and thus $\left\|\left(I-\pi_{h}\right) v\right\|=$ $\left\|\left(I-\pi_{h}\right) K \mathrm{~d} v_{h}\right\| \leq \eta\left\|\mathrm{d} v_{h}\right\|$. 
For the following estimates, let $P_{h}$ denote the $W$-orthogonal projection onto $V_{h}$, and for $w \in W$, define

$$
E(w)=\left\|\left(I-P_{h}\right) w\right\|=\inf _{v_{h} \in V_{h}}\left\|w-v_{h}\right\|,
$$

i.e., the best approximation to $w \in W$ by an element of $V_{h}$.

Theorem 3.6. $\left\|\mathrm{d}\left(u-u_{h}\right)\right\| \leq C E(\mathrm{~d} u)$.

Proof. Since $\mathrm{d} u_{h}=P_{\mathfrak{B}_{h}} f=P_{\mathfrak{B}_{h}} P_{\mathfrak{B}} f=P_{\mathfrak{B}_{h}} \mathrm{~d} u$ and $\pi_{h} \mathrm{~d} u \in \mathfrak{B}_{h}$, it follows that $\left\|\mathrm{d}\left(u-u_{h}\right)\right\|=\left\|\left(I-P_{\mathfrak{B}_{h}}\right) \mathrm{d} u\right\| \leq\left\|\left(I-\pi_{h}\right) \mathrm{d} u\right\| \leq C E(\mathrm{~d} u)$.

Theorem 3.7. $\left\|P_{\mathfrak{B}} u-P_{\mathfrak{B}_{h}} u_{h}\right\| \leq C\left(E\left(P_{\mathfrak{B}} u\right)+\eta[E(\mathrm{~d} u)+E(p)]\right)$.

Proof. We begin by writing

$$
\begin{aligned}
\left\|P_{\mathfrak{B}} u-P_{\mathfrak{B}_{h}} u_{h}\right\| & \leq\left\|P_{\mathfrak{B}} u-P_{\mathfrak{B}_{h}} P_{\mathfrak{B}} u\right\|+\left\|P_{\mathfrak{B}_{h}} P_{\mathfrak{B}} u-P_{\mathfrak{B}_{h}} u_{h}\right\| \\
& \leq\left\|\left(I-P_{\mathfrak{B}_{h}}\right) P_{\mathfrak{B}} u\right\|+\left\|P_{\mathfrak{B}_{h}}\left(u-u_{h}\right)\right\| .
\end{aligned}
$$

For the first term, optimality of $P_{\mathfrak{B}_{h}}$ and quasi-optimality of $\pi_{h}$, together with the fact that $\pi_{h} P_{\mathfrak{B}} u \in \mathfrak{B}_{h}$, implies

$$
\left\|\left(I-P_{\mathfrak{B}_{h}}\right) P_{\mathfrak{B}} u\right\| \leq\left\|\left(I-\pi_{h}\right) P_{\mathfrak{B}} u\right\| \leq C E\left(P_{\mathfrak{B}} u\right) .
$$

The remaining term will now be bounded using a duality-type argument.

Let $e=P_{\mathfrak{B}_{h}}\left(u-u_{h}\right), w=K e$, and $w_{h}=K_{h} e$. Since $e \in \mathfrak{B}_{h} \subset \mathfrak{B}$, we have $e=\mathrm{d} w=\mathrm{d} \pi_{h} w=\mathrm{d} w_{h}$. In particular, this implies that $\mathrm{d}\left(\pi_{h} w-w_{h}\right)=0$, so $\pi_{h} w-w_{h} \in \mathfrak{Z}_{h} \subset \mathfrak{Z}$ is orthogonal to both $w \in \mathfrak{B}^{*}$ and $w_{h} \in \mathfrak{B}_{h}^{*}$, and hence to $w-w_{h}$. Therefore, the Pythagorean theorem and Lemma 3.5 imply that

$$
\left\|w-w_{h}\right\| \leq\left\|\left(I-\pi_{h}\right) w\right\|=\left\|\left(I-\pi_{h}\right) K e\right\| \leq \eta\|e\| .
$$

Now, using $e=\mathrm{d} w_{h}$ and the variational principles (1) and (2), we have

$$
\|e\|^{2}=\left\langle e, \mathrm{~d} w_{h}\right\rangle=\left\langle u-u_{h}, \mathrm{~d} w_{h}\right\rangle=-\left\langle\mathrm{d}\left(u-u_{h}\right)+\left(p-p_{h}\right), w_{h}\right\rangle .
$$

Furthermore, since $w_{h} \in \mathfrak{B}_{h}^{*} \perp \mathfrak{H}_{h}$ and $w \in \mathfrak{B}^{*} \perp \mathfrak{Z}$, we can write this as

$$
\begin{aligned}
\|e\|^{2} & =-\left\langle\mathrm{d}\left(u-u_{h}\right)+\left(p-P_{\mathfrak{H}_{h}} p\right), w_{h}-w\right\rangle \\
& \leq\left[\left\|\mathrm{d}\left(u-u_{h}\right)\right\|+\left\|\left(I-P_{\mathfrak{H}_{h}}\right) p\right\|\right]\left\|w_{h}-w\right\| .
\end{aligned}
$$

We already have the estimate $\left\|\mathrm{d}\left(u-u_{h}\right)\right\| \leq C E(\mathrm{~d} u)$, while

$$
\left\|\left(I-P_{\mathfrak{H}_{h}}\right) p\right\|=\left\|\left(I-P_{\mathfrak{Z}_{h}}\right) p\right\| \leq\left\|\left(I-\pi_{h}\right) p\right\| \leq C E(p) .
$$

Combining these with $\left\|w-w_{h}\right\| \leq \eta\|e\|$ and dividing through by $\|e\|$, we finally obtain

which completes the proof.

$$
\|e\| \leq C \eta[E(\mathrm{~d} u)+E(p)]
$$

Theorem 3.8. $\left\|P_{\mathfrak{B}^{*}} u-P_{\mathfrak{B}_{h}^{*}} u_{h}\right\| \leq C\left[E\left(P_{\mathfrak{B}^{*}} u\right)+\eta E(\mathrm{~d} u)\right]$.

Proof. As in the previous proof, we begin by using the triangle inequality to split this into two pieces,

$$
\begin{aligned}
\left\|P_{\mathfrak{B}^{*}} u-P_{\mathfrak{B}_{h}^{*}} u_{h}\right\| & \leq\left\|P_{\mathfrak{B}^{*}} u-P_{\mathfrak{B}_{h}^{*}} P_{\mathfrak{B}^{*}} u\right\|+\left\|P_{\mathfrak{B}_{h}^{*}} P_{\mathfrak{B}^{*}} u-P_{\mathfrak{B}_{h}^{*}} u_{h}\right\| \\
& =\left\|\left(I-P_{\mathfrak{B}_{h}^{*}}\right) P_{\mathfrak{B}^{*}} u\right\|+\left\|P_{\mathfrak{B}_{h}^{*}}\left(P_{\mathfrak{B}^{*}} u-u_{h}\right)\right\| .
\end{aligned}
$$

Observe that, since $\mathfrak{Z}_{h} \subset \mathfrak{Z} \perp \mathfrak{B}^{*}$, it follows that

$$
P_{h} P_{\mathfrak{B}^{*}} u=P_{\mathfrak{Z}_{h}} P_{\mathfrak{B}^{*}} u+P_{\mathfrak{B}_{h}^{*}} P_{\mathfrak{B}^{*}} u=P_{\mathfrak{B}_{h}^{*}} P_{\mathfrak{B}^{*}} u .
$$


Therefore,

$$
\left\|\left(I-P_{\mathfrak{B}_{h}^{*}}\right) P_{\mathfrak{B}^{*}} u\right\|=\left\|\left(I-P_{h}\right) P_{\mathfrak{B}^{*}} u\right\|=E\left(P_{\mathfrak{B}^{*}} u\right) .
$$

For the second piece, let $v_{h}=P_{\mathfrak{B}_{h}^{*}}\left(\pi_{h} P_{\mathfrak{B}^{*}} u-u_{h}\right)$, so that

$$
\left\|P_{\mathfrak{B}_{h}^{*}}\left(P_{\mathfrak{B}^{*}} u-u_{h}\right)\right\| \leq\left\|P_{\mathfrak{B}_{h}^{*}}\left(I-\pi_{h}\right) P_{\mathfrak{B}^{*}} u\right\|+\left\|v_{h}\right\| \leq C E\left(P_{\mathfrak{B}^{*}} u\right)+\left\|v_{h}\right\| .
$$

It now suffices to control $\left\|v_{h}\right\|$.

To do so, we let $v=P_{\mathfrak{B}^{*}} v_{h}$, as in Lemma 3.5, and observe that

$$
\left\langle v, v_{h}\right\rangle=\left\langle v, v_{h}+P_{\mathfrak{Z}_{h}}\left(\pi_{h} P_{\mathfrak{B}^{*}} u-u_{h}\right)\right\rangle=\left\langle v, \pi_{h} P_{\mathfrak{B}^{*}} u-u_{h}\right\rangle .
$$

Therefore,

$$
\begin{aligned}
\left\|v_{h}\right\|^{2} & =\left\langle v_{h}-v, v_{h}\right\rangle+\left\langle v, v_{h}\right\rangle \\
& =\left\langle v_{h}-v, v_{h}\right\rangle+\left\langle v, \pi_{h} P_{\mathfrak{B}^{*}} u-u_{h}\right\rangle \\
& =\left\langle v_{h}-v, v_{h}\right\rangle+\left\langle v, \pi_{h} P_{\mathfrak{B}^{*}} u-P_{\mathfrak{B}^{*}} u\right\rangle+\left\langle v, P_{\mathfrak{B}^{*}} u-u_{h}\right\rangle .
\end{aligned}
$$

For the first two terms, Lemma 3.5 implies

$$
\begin{aligned}
\left\langle v_{h}-v, v_{h}\right\rangle+\left\langle v, \pi_{h} P_{\mathfrak{B}^{*}} u-P_{\mathfrak{B}^{*}} u\right\rangle & \leq\left\|v_{h}-v\right\|\left\|v_{h}\right\|+\|v\|\left\|\left(I-\pi_{h}\right) P_{\mathfrak{B}^{*}} u\right\| \\
& \leq\left[\eta\left\|\mathrm{d} v_{h}\right\|+C E\left(P_{\mathfrak{B}^{*}} u\right)\right]\left\|v_{h}\right\| .
\end{aligned}
$$

Now, $\mathrm{d} v_{h}=\pi_{h} \mathrm{~d} u-\mathrm{d} u_{h}$, so Theorem 3.6 implies that

$$
\left\|\mathrm{d} v_{h}\right\| \leq\left\|\left(I-\pi_{h}\right) \mathrm{d} u\right\|+\left\|\mathrm{d}\left(u-u_{h}\right)\right\| \leq C E(\mathrm{~d} u) .
$$

Finally, it remains to control $\left\langle v, u-u_{h}\right\rangle$. Since $v \in \mathfrak{B}^{*}$ and $P_{\mathfrak{B}^{*}}=K \mathrm{~d}$,

$$
\begin{aligned}
\left\langle v, u-u_{h}\right\rangle & =\left\langle K v, \mathrm{~d}\left(u-u_{h}\right)\right\rangle \\
& =\left\langle\left(I-\pi_{h}\right) K v, \mathrm{~d}\left(u-u_{h}\right)\right\rangle+\left\langle\pi_{h} K v, \mathrm{~d}\left(u-u_{h}\right)\right\rangle .
\end{aligned}
$$

For the first term, another application of Theorem 3.6 yields

$$
\left\langle\left(I-\pi_{h}\right) K v, \mathrm{~d}\left(u-u_{h}\right)\right\rangle \leq\left\|\left(I-\pi_{h}\right) K v\right\|\left\|\mathrm{d}\left(u-u_{h}\right)\right\| \leq C \eta E(\mathrm{~d} u)\left\|v_{h}\right\| .
$$

On the other hand, the second term vanishes: since $v \in \mathfrak{B}^{*}$, we have $K v \in \mathfrak{B}$ and $\pi_{h} K v \in \mathfrak{B}_{h}$, while $P_{\mathfrak{B}_{h}}\left(\mathrm{~d} u-\mathrm{d} u_{h}\right)=\mathrm{d} u_{h}-\mathrm{d} u_{h}=0$. Hence, we have finally shown that $\left\|v_{h}\right\| \leq C\left[E\left(P_{\mathfrak{B}^{*}} u\right)+\eta E(\mathrm{~d} u)\right]$, so the result follows.

Theorem 3.9. $\left\|p-p_{h}\right\| \leq C[E(p)+\mu E(\mathrm{~d} u)]$.

Proof. We begin by decomposing

$$
\left\|p-p_{h}\right\|^{2}=\left\|p-P_{\mathfrak{H}_{h}} p\right\|^{2}+\left\|P_{\mathfrak{H}_{h}} p-p_{h}\right\|^{2} .
$$

First, the inequality (3) implies that $\left\|p-P_{\mathfrak{H}_{h}} p\right\| \leq\left\|\left(I-\pi_{h}\right) p\right\| \leq C E(p)$. For the second term, we observe that $p=P_{\mathfrak{H}} f$, while $p_{h}=P_{\mathfrak{H}_{h}} f$. Therefore,

$$
p_{h}-P_{\mathfrak{H}_{h}} p=P_{\mathfrak{H}_{h}}\left(I-P_{\mathfrak{H}}\right) f=P_{\mathfrak{H}_{h}}\left(P_{\mathfrak{B}}+P_{\mathfrak{B}^{*}}\right) f=P_{\mathfrak{H}_{h}} P_{\mathfrak{B}} f=P_{\mathfrak{H}_{h}} \mathrm{~d} u,
$$

where we have used the fact that $P_{\mathfrak{H}_{h}} P_{\mathfrak{B}^{*}}=0$, since $\mathfrak{H}_{h} \subset \mathfrak{Z}_{h} \subset \mathfrak{Z} \perp \mathfrak{B}^{*}$. Now, using the orthogonality of the Hodge decomposition and the fact that $\pi_{h} \mathrm{~d} u=\mathrm{d} \pi_{h} u \in$ $\mathfrak{B}_{h} \subset \mathfrak{B}$, we get

$$
\begin{aligned}
\left\|P_{\mathfrak{H}_{h}} \mathrm{~d} u\right\|^{2} & =\left\langle\mathrm{d} u, P_{\mathfrak{H}_{h}} \mathrm{~d} u\right\rangle \\
& =\left\langle\left(I-\pi_{h}\right) \mathrm{d} u,\left(I-P_{\mathfrak{H}}\right) P_{\mathfrak{H}_{h}} \mathrm{~d} u\right\rangle \\
& \leq\left\|\left(I-\pi_{h}\right) \mathrm{d} u\right\|\left\|\left(I-P_{\mathfrak{H}}\right) P_{\mathfrak{H}_{h}} \mathrm{~d} u\right\| .
\end{aligned}
$$

Quasi-optimality of $\pi_{h}$ immediately implies $\left\|\left(I-\pi_{h}\right) \mathrm{d} u\right\| \leq C E(\mathrm{~d} u)$. On the other hand, since $P_{\mathfrak{H}_{h}} \mathrm{~d} u \in \mathfrak{Z}$, the Hodge decomposition implies that $\left(I-P_{\mathfrak{H}}\right) P_{\mathfrak{H}_{h}} \mathrm{~d} u \in \mathfrak{B}$. 
Hence, $\pi_{h}\left(I-P_{\mathfrak{H}}\right) P_{\mathfrak{H}_{h}} \mathrm{~d} u \in \mathfrak{B}_{h}$ is orthogonal to both $P_{\mathfrak{H}_{h}} \mathrm{~d} u \in \mathfrak{H}_{h}$ and $P_{\mathfrak{H}} P_{\mathfrak{H}_{h}} \mathrm{~d} u$, and thus to $\left(I-P_{\mathfrak{H}}\right) P_{\mathfrak{H}_{h}} \mathrm{~d} u$. Therefore, the Pythagorean theorem gives

$$
\begin{aligned}
\left\|\left(I-P_{\mathfrak{H}}\right) P_{\mathfrak{H}_{h}} \mathrm{~d} u\right\| & \leq\left\|\left(I-\pi_{h}\right)\left(I-P_{\mathfrak{H}}\right) P_{\mathfrak{H}_{h}} \mathrm{~d} u\right\| \\
& =\left\|\left(I-\pi_{h}\right) P_{\mathfrak{H}^{\prime}} P_{\mathfrak{H}_{h}} \mathrm{~d} u\right\| \\
& \leq \mu\left\|P_{\mathfrak{H}_{h}} \mathrm{~d} u\right\| .
\end{aligned}
$$

Finally, this shows that $\left\|P_{\mathfrak{H}_{h}} p-p_{h}\right\| \leq C \mu E(\mathrm{~d} u)$, so the proof is complete.

\section{Relationship to the Hodge-Laplace Problem}

In this section, we discuss the close relationship between the Hodge-Dirac problem and the Hodge-Laplace problem, which has been the main focus of much of the extant work on finite element exterior calculus (particularly Arnold et al. [3]). In fact, we show that some of the key estimates of finite element exterior calculus may be recovered as direct corollaries of the stability and convergence results presented in the previous two sections.

4.1. The Hodge-Laplace operator and mixed variational problem. The abstract Hodge-Laplace operator is $L=\mathrm{D}^{2}=\mathrm{dd}^{*}+\mathrm{d}^{*} \mathrm{~d}$, which is defined on the domain $\mathcal{D}(L)=\mathrm{D}^{-1}\left(V \cap V^{*}\right) \subset V \cap V^{*}$ and has kernel $\mathcal{N}(L)=\mathcal{N}(\mathrm{D})=\mathfrak{H}$. The Hodge-Laplace problem is then the following: Given $f \in W$, find $(u, p) \in$ $\left(\mathcal{D}(L) \cap \mathcal{N}(L)^{\perp}\right) \oplus \mathcal{N}(L)$ such that

$$
L u+p=f .
$$

To solve this, we may simply solve the Hodge-Dirac problem $\mathrm{D} w+p=f$, and then solve another Hodge-Dirac problem $\mathrm{D} u=w$. (Since $w \perp \mathfrak{H}$, the harmonic part is omitted from the second problem.) Therefore,

$$
L u+p=\mathrm{D}^{2} u+p=\mathrm{D}(\mathrm{D} u)+p=\mathrm{D} w+p=f,
$$

so $(u, p)$ is indeed a solution to the Hodge-Laplace problem, as claimed.

To apply finite-element techniques to this problem, it must be put into a variational form. Naively, we might think to use the variational problem: Find $(u, p) \in(V \cap$ $\left.V^{*}\right) \times \mathfrak{H}$ such that

$$
\begin{aligned}
\left\langle\mathrm{d}^{*} u, \mathrm{~d}^{*} v\right\rangle+\langle\mathrm{d} u, \mathrm{~d} v\rangle+\langle p, v\rangle & =\langle f, v\rangle, & & \forall v \in V \cap V^{*}, \\
\langle u, q\rangle & =0, & & \forall q \in \mathfrak{H} .
\end{aligned}
$$

This problem is easily shown to be well-posed, by virtue of the boundedness, symmetry, and coercivity of the bilinear form $(u, p ; v, q) \mapsto\left\langle\mathrm{d}^{*} u, \mathrm{~d}^{*} v\right\rangle+\langle\mathrm{d} u, \mathrm{~d} v\rangle+\langle p, v\rangle+\langle u, q\rangle$. In fact, the bilinear form gives an equivalent inner product for the Hilbert space $\left(V \cap V^{*}\right) \times \mathfrak{H}$, so this is a simple application of the Riesz representation theorem to the functional $(v, q) \mapsto\langle f, v\rangle$. Yet, despite the stability of this formulation, it is generally unsuitable for the finite element method due to consistency difficulties. In particular, for the case of the Hodge-de Rham complex, piecewise polynomial differential forms are too regular to approximate singular elements of $V \cap V^{*}$, cf. Brezzi and Fortin [12, Arnold et al. [3].

Instead, it is preferable to use the following mixed variational formulation: Find $(\sigma, u, p) \in V \times V \times \mathfrak{H}$ such that

$$
\begin{aligned}
\langle\sigma, \tau\rangle-\langle u, \mathrm{~d} \tau\rangle & =0, & & \forall \tau \in V, \\
\langle\mathrm{~d} \sigma, v\rangle+\langle\mathrm{d} u, \mathrm{~d} v\rangle+\langle p, v\rangle & =\langle f, v\rangle, & & \forall v \in V, \\
\langle u, q\rangle & =0, & & \forall q \in \mathfrak{H} .
\end{aligned}
$$


Indeed, if $(\sigma, u, p)$ is a solution, then the first line of (5) implies $\sigma=\mathrm{d}^{*} u$, while the second line implies that

$$
L u+p=\mathrm{dd}^{*} u+\mathrm{d}^{*} \mathrm{~d} u+p=\mathrm{d} \sigma+\mathrm{d}^{*} \mathrm{~d} u+p=f .
$$

(Note that $\mathrm{d} \sigma+\mathrm{d}^{*} \mathrm{~d} u+p=f$ is precisely the Hodge decomposition of $f$.) We now show that - as with the non-variational form of the problems - we may solve the Hodge-Laplace mixed variational problem (5) by simply solving the Hodge-Dirac problem (1) twice.

Theorem 4.1. If $(w, p) \in V \times \mathfrak{H}$ solves the Hodge-Dirac variational problem for $f$, and $(u, 0) \in V \times \mathfrak{H}$ solves the Hodge-Dirac variational problem for $w$, then $(w-\mathrm{d} u, u, p) \in V \times V \times \mathfrak{H}$ solves (5).

Proof. For any $\tau \in V$, we have

$$
\langle w-\mathrm{d} u, \tau\rangle-\langle u, \mathrm{~d} \tau\rangle=\langle w, \tau\rangle-(\langle\mathrm{d} u, \tau\rangle+\langle u, \mathrm{~d} \tau\rangle)=\langle w, \tau\rangle-\langle w, \tau\rangle=0 .
$$

Next, for any $v \in V$, we have

$$
\begin{aligned}
\langle\mathrm{d}(w-\mathrm{d} u), v\rangle+\langle\mathrm{d} u, \mathrm{~d} v\rangle+\langle p, v\rangle & =\langle\mathrm{d} w, v\rangle+\langle\mathrm{d} u, \mathrm{~d} v\rangle+\langle p, v\rangle \\
& =\langle\mathrm{d} w, v\rangle+(\langle\mathrm{d} u, \mathrm{~d} v\rangle+\langle u, \mathrm{~d} \mathrm{~d} v\rangle)+\langle p, v\rangle \\
& =\langle\mathrm{d} w, v\rangle+\langle w, \mathrm{~d} v\rangle+\langle p, v\rangle \\
& =\langle f, v\rangle .
\end{aligned}
$$

Finally, since $(u, 0) \in V \times \mathfrak{H}$ solves the Hodge-Dirac variational problem for $w$, we have $\langle u, q\rangle=0$ for all $q \in \mathfrak{H}$, which completes the proof.

As an immediate consequence of this fact, together with the well-posedness result Corollary 2.5 for Hodge-Dirac, we obtain well-posedness for Hodge-Laplace. (Compare Arnold et al. [3, Theorem 3.1].)

Theorem 4.2. The mixed variational problem for the abstract Hodge-Laplace operator is well-posed. That is, there exists a constant $c^{\prime}$, depending only on the Poincaré constant $c_{P}$, such that for all $f \in W$, the problem (5) has a unique solution $(\sigma, u, p) \in V \times \mathfrak{H}$, which satisfies the estimate

$$
\|\sigma\|_{V}+\|u\|_{V}+\|p\| \leq c^{\prime}\|f\| .
$$

Proof. By Theorem 4.1 and the triangle inequality,

$$
\|\sigma\|_{V}+\|u\|_{V}+\|p\|=\|w-\mathrm{d} u\|_{V}+\|u\|_{V}+\|p\| \leq\|w\|_{V}+2\|u\|_{V}+\|p\| .
$$

Now, since $(u, 0)$ solves the Hodge-Dirac problem for $w$, Corollary 2.5 implies that $\|u\|_{V} \leq c\|w\| \leq c\|w\|_{V}$, where $c$ depends only on $c_{P}$. Therefore,

$$
\|\sigma\|_{V}+\|u\|_{V}+\|p\| \leq\|w\|_{V}+2 c\|w\|_{V}+\|p\| \leq(1+2 c)\left(\|w\|_{V}+\|p\|\right) .
$$

Another application of Corollary 2.5 gives $\|w\|_{V}+\|p\| \leq c\|f\|$, so

$$
\|\sigma\|_{V}+\|u\|_{V}+\|p\| \leq(1+2 c) c\|f\|=c^{\prime}\|f\|
$$

which completes the proof.

While we have shown that one can solve the Hodge-Laplace problem by solving related Hodge-Dirac problems, the converse is also true: if $L u+p=f$, then clearly $\mathrm{D}(\mathrm{D} u)+p=f$, so $(\mathrm{D} u, p)$ solves the Hodge-Dirac problem. (Note that $\mathrm{D} u \perp \mathfrak{H}$, since the range of $\mathrm{D}$ is $\mathfrak{B} \oplus \mathfrak{B}^{*}=\mathfrak{H}^{\perp}$.) A similar result holds for the variational problems, as we now show. 
Theorem 4.3. If $(\sigma, u, p) \in V \times V \times \mathfrak{H}$ solves (5), then $(\sigma+\mathrm{d} u, p) \in V \times \mathfrak{H}$ solves (1).

Proof. For any $v \in V$, since $\mathrm{d} d u=0$, we have $\langle\mathrm{d}(\sigma+\mathrm{d} u), v\rangle=\langle\mathrm{d} \sigma, v\rangle$. Similarly, by the first line of (5) with $\tau=\mathrm{d} v$, we have $\langle\sigma, \mathrm{d} v\rangle=\langle u, \mathrm{~d} d v\rangle=0$, so $\langle\sigma+\mathrm{d} u, \mathrm{~d} v\rangle=$ $\langle\mathrm{d} u, \mathrm{~d} v\rangle$. Therefore, by the second line of (5),

$$
\langle\mathrm{d}(\sigma+\mathrm{d} u), v\rangle+\langle\sigma+\mathrm{d} u, \mathrm{~d} v\rangle+\langle p, v\rangle=\langle\mathrm{d} \sigma, v\rangle+\langle\mathrm{d} u, \mathrm{~d} v\rangle+\langle p, v\rangle=\langle f, v\rangle .
$$

Finally, for any $q \in \mathfrak{H}$, the first line of (5) gives $\langle\sigma, q\rangle=\langle u, \mathrm{~d} q\rangle=0$, since $\mathfrak{H} \subset \mathfrak{Z}$. Furthermore, we also have $\langle\mathrm{d} u, q\rangle=0$, since $\mathfrak{H} \perp \mathfrak{B}$. Hence,

$$
\langle\sigma+\mathrm{d} u, q\rangle=0
$$

which completes the proof.

4.2. The discrete Hodge-Laplace problem. Again, let $V_{h} \subset V$ be a closed subspace satisfying the conditions discussed in Section 3 (i.e., $\mathrm{d}_{h}=\left.\mathrm{d}\right|_{V_{h}}$ is a closednilpotent operator on $V_{h}$ ) and equipped with a bounded commuting projection $\pi_{h}$. We then consider the discrete Hodge-Laplace mixed variational problem: Find $\left(\sigma_{h}, u_{h}, p_{h}\right) \in V_{h} \times V_{h} \times \mathfrak{H}_{h}$ such that

$$
\begin{aligned}
\left\langle\sigma_{h}, \tau_{h}\right\rangle-\left\langle u_{h}, \mathrm{~d} \tau_{h}\right\rangle & =0, & & \forall \tau_{h} \in V_{h}, \\
\left\langle\mathrm{~d} \sigma_{h}, v_{h}\right\rangle+\left\langle\mathrm{d} u_{h}, \mathrm{~d} v_{h}\right\rangle+\left\langle p_{h}, v_{h}\right\rangle & =\left\langle f, v_{h}\right\rangle, & & \forall v_{h} \in V_{h}, \\
\left\langle u_{h}, q_{h}\right\rangle & =0, & & \forall q_{h} \in \mathfrak{H}_{h} .
\end{aligned}
$$

Applying Theorem 4.1 to $V_{h}$ implies that this discrete Hodge-Laplace problem can be solved by solving two discrete Hodge-Dirac problems (2) sequentially. That is, if $\left(w_{h}, p_{h}\right) \in V_{h} \times \mathfrak{H}_{h}$ solves the discrete Hodge-Dirac problem for $f$, and $\left(u_{h}, 0\right) \in$ $V_{h} \times \mathfrak{H}_{h}$ solves the discrete Hodge-Dirac problem for $w_{h}$, then $\left(w_{h}-\mathrm{d} u_{h}, u_{h}, p_{h}\right) \in$ $V_{h} \times V_{h} \times \mathfrak{H}_{h}$ solves (6). Thus, as in Theorem 4.2, the discrete Hodge-Laplace problem is well-posed, and moreover Corollary 3.3 implies that the constant $c_{h}^{\prime}$ depends only on $c_{P}$ and $\left\|\pi_{h}\right\|$.

As a result, it is now straightfoward to obtain an error estimate for the approximation of the Hodge-Laplace problem (5) by the discrete problem (6), which follows from the analogous Hodge-Dirac result, Theorem 3.4. (Compare Arnold et al. 3, Theorem 3.9].)

Theorem 4.4. Under the hypotheses of Theorem 3.4, if $(\sigma, u, p) \in V \times V \times \mathfrak{H}$ solves (5) and $\left(\sigma_{h}, u_{h}, p_{h}\right) \in V_{h} \times V_{h} \times \mathfrak{H}_{h}$ solves (6), then we have the error estimate

$$
\begin{aligned}
\| \sigma- & \sigma_{h}\left\|_{V}+\right\| u-u_{h}\left\|_{V}+\right\| p-p_{h} \| \\
& \leq C\left(\inf _{\tau \in V_{h}}\|\sigma-\tau\|_{V}+\inf _{v \in V_{h}}\|u-v\|_{V}+\inf _{q \in V_{h}}\|p-q\|_{V}+\mu \inf _{v \in V_{h}}\left\|P_{\mathfrak{B}} u-v\right\|_{V}\right) .
\end{aligned}
$$

Proof. By Theorem 4.1, we have $\sigma=w-\mathrm{d} u$ and $\sigma_{h}=w_{h}-\mathrm{d} u_{h}$, where $(w, p)$ and $\left(w_{h}, p_{h}\right)$ solve, respectively, the Hodge-Dirac and discrete Hodge-Dirac problems for $f$. Therefore, by the triangle inequality,

$$
\left\|\sigma-\sigma_{h}\right\|_{V}+\left\|u-u_{h}\right\|_{V}+\left\|p-p_{h}\right\| \leq C\left(\left\|w-w_{h}\right\|_{V}+\left\|u-u_{h}\right\|_{V}+\left\|p-p_{h}\right\|\right) .
$$

Now, by Theorem 3.4.

$$
\left\|w-w_{h}\right\|_{V}+\left\|p-p_{h}\right\| \leq C\left(\inf _{v \in V_{h}}\|w-v\|_{V}+\inf _{q \in V_{h}}\|p-q\|_{V}+\mu \inf _{v \in V_{h}}\left\|P_{\mathfrak{B}} w-v\right\|_{V}\right) .
$$


Writing $w=\sigma+\mathrm{d} u$, we then get

$$
\inf _{v \in V_{h}}\|w-v\|_{V} \leq \inf _{\tau \in V_{h}}\|\sigma-\tau\|_{V}+\inf _{v \in V_{h}}\|u-v\|_{V} .
$$

Moreover, the first line of the Hodge-Laplace variational principle (5) implies that $P_{\mathfrak{B}} \sigma=0$, so $P_{\mathfrak{B}} w=\mathrm{d} u$, and thus

$$
\inf _{v \in V_{h}}\left\|P_{\mathfrak{B}} w-v\right\|_{V} \leq \inf _{v \in V_{h}}\|u-v\|_{V},
$$

Altogether, we now have

$$
\left\|w-w_{h}\right\|_{V}+\left\|p-p_{h}\right\| \leq C\left(\inf _{\tau \in V_{h}}\|\sigma-\tau\|_{V}+\inf _{v \in V_{h}}\|u-v\|_{V}+\inf _{q \in V_{h}}\|p-q\|_{V}\right) .
$$

so it suffices to control the remaining term $\left\|u-u_{h}\right\|_{V}$.

Recall that $(u, 0)$ solves the Hodge-Dirac problem for $w$, while $\left(u_{h}, 0\right)$ solves the Hodge-Dirac problem for $w_{h}$. Since the right-hand sides of these problems are different, it is not possible to apply Theorem 3.4 just yet: there is a "variational crime" that must be controlled. To do so, let $\left(u_{h}^{\prime}, P_{\mathfrak{H}_{h}} w\right)$ be the solution of the discrete Hodge-Dirac problem for $w$, so using the triangle inequality,

$$
\left\|u-u_{h}\right\|_{V} \leq\left\|u-u_{h}^{\prime}\right\|_{V}+\left\|u_{h}^{\prime}-u_{h}\right\|_{V} .
$$

By Corollary 3.3, the discrete well-posedness result, we have $\left\|u_{h}^{\prime}-u_{h}\right\|_{V} \leq C\left\|w-w_{h}\right\|$, which is already under control, while another application of Theorem 3.4 gives

$$
\left\|u-u_{h}^{\prime}\right\|_{V} \leq\left\|u-u_{h}^{\prime}\right\|_{V}+\left\|P_{\mathfrak{H}_{h}} w\right\| \leq C\left(\inf _{v \in V_{h}}\|u-v\|_{V}+\inf _{v \in V_{h}}\left\|P_{\mathfrak{B}} u-v\right\|_{V}\right) .
$$

Finally, combining these estimates yields the claimed result.

\section{A note on Hilbert COMPleXes AND Nilpotent operators}

The framework of finite element exterior calculus, as developed by Arnold et al. [3], is described using Hilbert complexes (cf. Brüning and Lesch [13]), which are closely related to the nilpotent operators considered here (and also by Axelsson and McIntosh [6], Axelsson et al. [5]). Rather than a single Hilbert space $W$ and nilpotent operator d, a Hilbert complex consists of a sequence of several Hilbert spaces $W^{k}$ and closed, densely-defined operators $\mathrm{d}^{k}: V^{k} \subset W^{k} \rightarrow V^{k+1} \subset W^{k+1}$ satisfying the nilpotency property $\mathrm{d}^{k} \mathrm{~d}^{k-1}=0$. The domains and operators thus form a cochain complex (called the domain complex) in the category of Hilbert spaces, as depicted in the following diagram:

$$
\cdots V^{k-1} \stackrel{\mathrm{d}^{k-1}}{\longrightarrow} V^{k} \stackrel{\mathrm{d}^{k}}{\longrightarrow} V^{k+1} \cdots .
$$

It follows immediately that $\mathrm{d}=\bigoplus_{k} \mathrm{~d}^{k}$ is a nilpotent operator on $W=\bigoplus_{k} W^{k}$, with dense domain $V=\bigoplus_{k} V^{k}$. In other words, a Hilbert complex is simply a graded Hilbert space with a graded nilpotent operator. Conversely, if $W$ is a Hilbert space with a nilpotent operator d, then it corresponds to the Hilbert complex with $W^{k}=W$ and $\mathrm{d}^{k}=\mathrm{d}$ for all $k \in \mathbb{Z}$, i.e., its domain complex has the infinite diagram

$$
\cdots V \stackrel{\mathrm{d}}{\rightarrow} V \stackrel{\mathrm{d}}{\rightarrow} V \cdots .
$$

Additionally, Arnold et al. [3] define some additional structures that a Hilbert complex may have, corresponding to the different types of nilpotent operators defined in Definition 2.1. A "dictionary" between nilpotent operator terminology and that of Hilbert complexes is given in Table 1 . 


$\begin{aligned} \text { ungraded } & \text { graded } \\ \text { nilpotent operator } & \text { Hilbert complex } \\ \text { closed-nilpotent operator } & \text { closed Hilbert complex } \\ \text { Fredholm-nilpotent operator } & \text { Fredholm complex } \\ \text { diffuse Fredholm-nilpotent operator } & \text { Hilbert complex with } \\ & \text { the "compactness property" }\end{aligned}$

TABLE 1. A "dictionary" between the language of nilpotent operators and that of Hilbert complexes.

To discretize the Hodge-Laplace problem on a closed Hilbert complex, Arnold et al. [3] consider a Hilbert subcomplex consisting of closed subspaces $V_{h}^{k} \subset V^{k}$ such that $\mathrm{d}_{h}^{k}=\left.\mathrm{d}^{k}\right|_{V_{h}^{k}}$, and equipped with bounded commuting projections $\pi_{h}^{k}: V^{k} \rightarrow V_{h}^{k}$. Again, taking the direct sums $V_{h}=\bigoplus_{k} V_{h}^{k}, \mathrm{~d}_{h}=\bigoplus_{k} \mathrm{~d}_{h}^{k}$, and $\pi_{h}=\bigoplus_{k} \pi_{h}^{k}$, one gets an approximating subspace and bounded commuting projection satisfying the conditions of Section 3. In other words, a Hilbert subcomplex with bounded commuting projections is precisely the situation of Section 3 , in the case where the spaces and maps are graded.

This observation implies that the elements commonly used in finite element exterior calculus also yield a stable discretization of the Hodge-Dirac problem. Indeed, these elements - which include the $\mathcal{P}_{r}$ and $\mathcal{P}_{r}^{-}$families of piecewise-polynomial differential forms on simplicial meshes (cf. Arnold et al. [2, 3]) and the more recent $\mathcal{S}_{r}$ family on cubical meshes (cf. Arnold and Awanou [1]) - give subcomplexes of the $L^{2}$ de Rham complex with bounded commuting projections. Therefore, taking the direct sum over all degrees $k$, one obtains subspaces and projections satisfying the conditions in Section 3, implying stability and convergence for the Hodge-Dirac problem.

\section{Numerical APPLICATION: COMPUTING VECTOR FIELDS WITH PRESCRIBED DIVERGENCE AND CURL}

We conclude with a simple numerical example, illustrating an application of the techniques developed throughout the paper.

Given a bounded domain $U \subset \mathbb{R}^{2}$, let $L^{2} \Omega(U)$ be the $L^{2}$ de Rham complex on $U$. For the domain of d, take either the domain complex $H \Omega(U)$, with natural boundary conditions, or $\stackrel{\circ}{H} \Omega(U)$, with essential boundary conditions, i.e., 0 -forms vanish on the boundary and 1-forms vanish tangent to the boundary (cf. Arnold et al. 3, Sections 4.2 and 6.2]). Now, given scalar functions $f, g \in L^{2}(U)$, consider the Hodge-Dirac problem

$$
\mathrm{D} u+p=f+g \mathrm{~d} x_{1} \wedge \mathrm{d} x_{2} .
$$

Notice that, since there is no 1-form component on the right-hand side, the 0 - and 2 -form components of $u$ vanish, so $u$ is simply a 1-form. Furthermore, if $f$ and $g$ are orthogonal to harmonic 0 - and 2-forms, respectively, then $p=0$ as well. If, in addition, $U$ is simply connected (so that there are no harmonic 1-forms), then $u$ is the unique 1 -form satisfying

$$
\mathrm{d}^{*} u=f, \quad \mathrm{~d} u=g \mathrm{~d} x_{1} \wedge \mathrm{d} x_{2} .
$$

Identifying the 1 -form $u=u_{1} \mathrm{~d} x_{1}+u_{2} \mathrm{~d} x_{2}$ with the vector field $\left(u_{1}, u_{2}\right)$, this is equivalent to

$$
-\operatorname{div} u=f, \quad \operatorname{curl} u=g .
$$



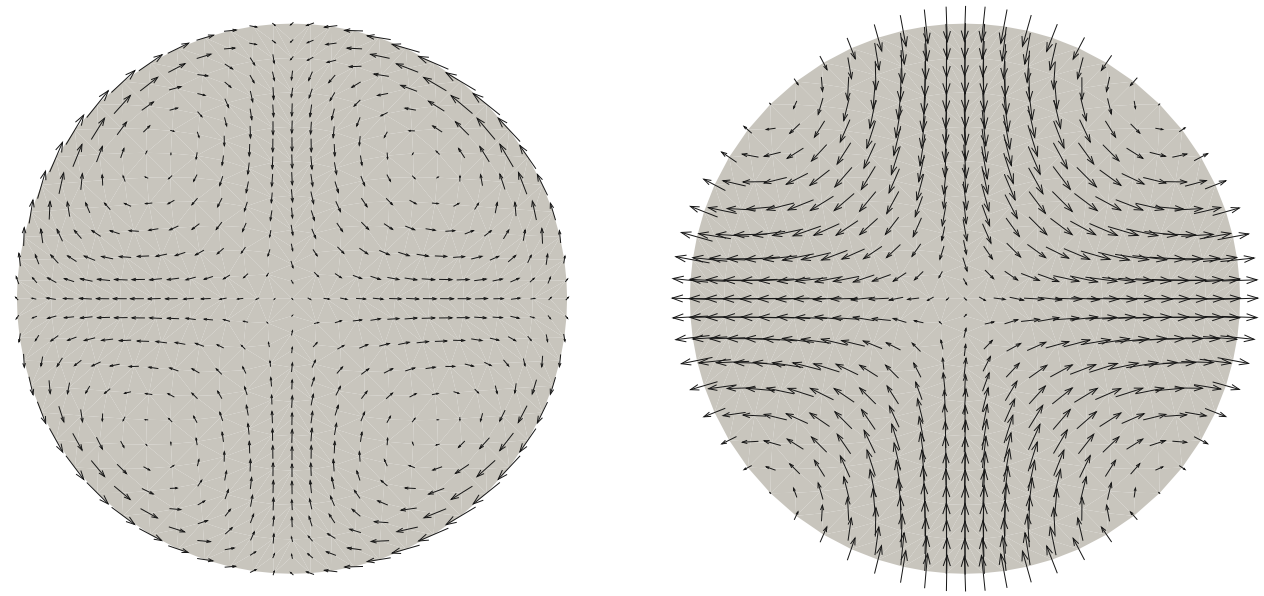

FiguRE 1. Divergence-free vector fields on the unit disk with curl equal to $x_{1} x_{2}$. Left: natural boundary conditions (vanishing normal component). Right: essential boundary conditions (vanishing tangential component).

That is, the Hodge-Dirac problem allows us to find a vector field with prescribed divergence and curl, subject to either natural boundary conditions ( $u$ is tangent to the boundary) or essential boundary conditions ( $u$ is normal to the boundary).

Figure 1 shows numerical solutions to this problem for both choices of boundary conditions, where $U$ is taken to be the unit disk, $f=0$, and $g=x_{1} x_{2}$. (Since $\mathfrak{H}$ contains only constant functions, and $\int_{U} f=\int_{U} g=0$, it follows that each is orthogonal to $\mathfrak{H}$.) The discrete Hodge-Dirac problem (2) was implemented using FEniCS (Logg et al. [30]), where $V_{h}$ was taken to be the space of $\mathcal{P}_{1}^{-}$differential forms, i.e., Whitney forms.

Finally, we note that this problem of "designing" vector fields has an interesting application in computer graphics, as studied by Fisher et al. 24]. For hair- and fur-like textures, rather than having an artist painstakingly specify the direction of every individual hair, it is easier for the artist to specify the divergence and curl at a set of control points (e.g., cowlicks, whorls), and to solve for the vector field of hair orientations satisfying the desired properties.

\section{Conclusion}

We have shown that the general techniques of finite element exterior calculus, in addition to their previously-studied application to the abstract Hodge-Laplace problem, may also be applied to an appropriately defined abstract Hodge-Dirac problem. In addition to resolving questions in the Clifford analysis community about the discretization of certain Dirac-type operators, this also yields new insight into the stability and convergence estimates of Arnold et al. 3], which may be recovered as corollaries of the estimates obtained here. Finally, due to the direct relationship between Hilbert complexes and nilpotent operators, it follows that stable elements for the Hodge-Laplace problem are also stable for the Hodge-Dirac problem, and hence no new or exotic elements need to be introduced.

Acknowledgments. P. L. gratefully acknowledges the support of the Australian Research Council, the Australian Mathematical Sciences Institute, and the Australian National University. A. S. gratefully acknowledges the support of the Simons 
Foundation through its AMS-Simons Travel Grant and Collaboration Grants in Mathematics programs.

\section{REFERENCES}

[1] D. N. Arnold And G. Awanou, Finite element differential forms on cubical meshes, 2013. To appear in Math. Comp.

[2] D. N. Arnold, R. S. FAlk, And R. Winther, Finite element exterior calculus, homological techniques, and applications, Acta Numer., 15 (2006), pp. 1-155.

[3] — Finite element exterior calculus: from Hodge theory to numerical stability, Bull. Amer. Math. Soc. (N.S.), 47 (2010), pp. 281-354.

[4] M. F. АтіYAн And I. M. Singer, The index of elliptic operators on compact manifolds, Bull. Amer. Math. Soc., 69 (1963), pp. 422-433.

[5] A. Axelsson, S. Keith, And A. McIntosh, Quadratic estimates and functional calculi of perturbed Dirac operators, Invent. Math., 163 (2006), pp. 455-497.

[6] A. Axelsson And A. McIntosh, Hodge decompositions on weakly Lipschitz domains, in Advances in analysis and geometry, Trends Math., Birkhäuser, Basel, 2004, pp. 3-29.

[7] I. BABUŠKA, Error-bounds for finite element method, Numer. Math., 16 (1971), pp. 322-333.

[8] P. B. Bochev and J. M. Hyman, Principles of mimetic discretizations of differential operators, in Compatible spatial discretizations, vol. 142 of IMA Vol. Math. Appl., Springer, New York, 2006, pp. 89-119.

[9] A. Bossavit, Whitney forms: a class of finite elements for three-dimensional computations in electromagnetism, Science, Measurement and Technology, IEE Proceedings A, 135 (1988), pp. 493-500.

[10] - Computational electromagnetism, Electromagnetism, Academic Press Inc., San Diego, CA, 1998. Variational formulations, complementarity, edge elements.

[11] F. Brackx, H. De Schepper, F. Sommen, and L. Van de Voorde, Discrete Clifford analysis: a germ of function theory, in Hypercomplex analysis, Trends Math., Birkhäuser Verlag, Basel, 2009, pp. 37-53.

[12] F. Brezzi and M. Fortin, Mixed and hybrid finite element methods, vol. 15 of Springer Series in Computational Mathematics, Springer-Verlag, New York, 1991.

[13] J. Brüning And M. Lesch, Hilbert complexes, J. Funct. Anal., 108 (1992), pp. 88-132.

[14] S. H. Christiansen And R. Winther, Smoothed projections in finite element exterior calculus, Math. Comp., 77 (2008), pp. 813-829.

[15] P. G. Ciarlet, The finite element method for elliptic problems, North-Holland Publishing Co., Amsterdam, 1978. Studies in Mathematics and its Applications, Vol. 4.

[16] J. CNOPS, An introduction to Dirac operators on manifolds, vol. 24 of Progress in Mathematical Physics, Birkhäuser Boston Inc., Boston, MA, 2002.

[17] R. Delanghe, Clifford analysis: history and perspective, Comput. Methods Funct. Theory, 1 (2001), pp. 107-153.

[18] M. Desbrun, A. N. Hirani, M. Leok, and J. E. Marsden, Discrete exterior calculus, 2005. Preprint.

[19] P. A. M. Dirac, The quantum theory of the electron, Proc. R. Soc. Lond. A, 117 (1928), pp. 610-624.

[20] M. G. Eastwood And J. Ryan, Aspects of Dirac operators in analysis, Milan J. Math., 75 (2007), pp. 91-116.

[21] R. S. FAlK And R. Winther, Local bounded cochain projections, 2013. To appear in Math. Comp.

[22] N. Faustino, Discrete Clifford analysis, Ph.D. thesis, Universidade de Aveiro, 2009.

[23] N. Faustino, U. Kähler, And F. Sommen, Discrete Dirac operators in Clifford analysis, Adv. Appl. Clifford Algebr., 17 (2007), pp. 451-467.

[24] M. Fisher, P. Schröder, M. Desbrun, And H. Hoppe, Design of tangent vector fields, ACM Trans. Graph., 26 (2007).

[25] T. Friedrich, Dirac operators in Riemannian geometry, vol. 25 of Graduate Studies in Mathematics, American Mathematical Society, Providence, RI, 2000. Translated from the 1997 German original by Andreas Nestke.

[26] J. Harrison, Ravello lecture notes on geometric calculus-Part I, 2005. Preprint.

[27] R. Hiptmair, Canonical construction of finite elements, Math. Comp., 68 (1999), pp. 1325-1346.

[28] — Finite elements in computational electromagnetism, Acta Numer., 11 (2002), pp. 237-339. 
[29] P. R. Kotiuga, Hodge decompositions and computational electromagnetics, Ph.D. thesis, McGill University, 1984.

[30] A. Logg, K.-A. Mardal, G. N. Wells, et Al., Automated Solution of Differential Equations by the Finite Element Method, Springer, 2012.

[31] G. Moisil and N. Theodoresco, Fonctions holomorphes dans l'espace., Mathematica (Cluj), 5 (1931), pp. $142-159$.

[32] J.-C. NÉDÉLEC, Mixed finite elements in $\mathbb{R}^{3}$, Numer. Math., 35 (1980), pp. 315-341.

[33] — A new family of mixed finite elements in $\mathbb{R}^{3}$, Numer. Math., 50 (1986), pp. 57-81.

[34] E. Witten, A new proof of the positive energy theorem, Comm. Math. Phys., 80 (1981), pp. 381-402.

University of Newcastle, Callaghan, NSW, Australia

E-mail address: paul.leopardi@newcastle.edu.au

Washington University, St. Louis, Missouri, USA

E-mail address: astern@math.wustl.edu 